\title{
EL PROCESO DE GESTIÓN Y EL DESEMPEÑO ORGANIZACIONAL Una aproximación a la nueva gestión pública desde el ámbito de los gobiernos locales
}

\author{
THE MANAGEMENT PROCESSES AND ORGANIZATIONAL PERFORMANCE \\ A new approach into public management from the \\ perspective of local governments
}

\author{
Carlos del Castillo ${ }^{1}$ y Braulio Vargas ${ }^{2}$
}

\begin{abstract}
RESUMEN
La medición del desempeño de la organización, en particular la búsqueda de un modelo que acerque el proceso de gestión a los resultados esperados a través de estrategias, ha sido uno de los temas más importantes de la Administración en las últimas décadas. Recientemente, se han hecho esfuerzos por adaptar a las organizaciones del sector público enfoques y tecnologías inicialmente desarrollados para las empresas, sobre todo en países que, como el Perú, buscan incidir en la capacidad de los gobiernos para producir resultados. Empero, la evidencia concreta de los gobiernos locales sugiere que la implementación de sistemas de gestión por procesos en este ámbito ve reducida su expectativa de éxito por la naturaleza altamente estructurada de la gestión, la rigidez del diseño organizacional y la propia normativa gubernamental. Este artículo plantea que la mejora del rendimiento deviene de conciliar las dimensiones de la estrategia y la estructura. Sobre esa base, se presenta una herramienta para estimar el desempeño organizacional en función de flexibilizar la estructura funcional y adaptarla a una lógica de gestión por procesos. Así es más factible que la estrategia gane intensidad, provoque la alineación de los procesos y visibilice los resultados de estos, permitiendo evaluar el desempeño de las áreas, los gestores y, en definitiva, la organización.
\end{abstract}

Palabras claves: gestión pública, gobiernos locales, medición del desempeño, modelos de gestión, indicadores.

\begin{abstract}
The measurement of organizational performance, in particular the search for a model that brings the management process together with the expected results through strategies, has been one of the most important issues in the Administration in recent decades. Recently, efforts have been made to adapt to public sector organizations approaches and technologies originally developed for businesses, especially in countries such as Peru, seeking to influence governments' ability to produce results.

However, the concrete evidence of local government suggests that the implementation of management processes in this area reduces its chances of success because of the highly structured nature of management, the rigidity of the organizational design and the government regulation itself. This article argues that the performance improvement comes from reconciling the dimensions of the strategy and the structure. On this basis, there is a tool to assess organizational performance in terms of make flexible the functional structure and to adapt it towards logic of process management. In this way the strategy may gain intensity, causing the alignment of processes and create visible results of these, allowing to evaluate the performance of the areas, managers and ultimately, the organization.
\end{abstract}

Key words: Public management, local government, performance measurement, management models, indicators.

1. Universidad ESAN (Perú). Magíster en Administración de la Universidad ESAN. <cdelcastillo@esan.edu.pe>.

2. Universidad ESAN (Perú). Magíster en Administración de la Universidad ESAN. <bvargas@esan.edu.pe>. 


\section{INTRODUCCIÓN Y MARCO CONCEPTUAL}

Durante las últimas cuatro décadas, la búsqueda de un modelo de gestión que comprenda la evaluación del desempeño de la organización ha alimentado las expectativas de los gestores en organizaciones públicas y privadas. Los esfuerzos para hallar el ansiado modelo han ido desde la implementación de sistemas rígidos y altamente estructurados, en los que los procedimientos han sido el eje de la gestión, hasta la adopción de sistemas flexibles, en los que la medida de proximidad a los resultados esperados y la adición del potencial humano a la ventaja competitiva de la organización son los ejes que orientan el proceso conocido como la «gestión» (Quinn, Farman, Thompson y McGrath, 2003; Ansoff y McDonnell, 1990).

En este sentido, la gestión requiere entenderse como un proceso que existe explícita e implícitamente en la medida en que las estrategias y las medidas de logro de objetivos y metas han sido definidas, desde el corto hacia el largo plazo. A ello se debe añadir que la puesta en marcha del proceso de gestión en condiciones reales también demanda desarrollos completamente nuevos en campos como el proceso estratégico, la firma y los límites de la firma, los costos de agencia y los costos de transacción, la generación de valor (para el accionista y el cliente) y su relación con el desempeño en el nivel de la firma (Becerra, 2009); además de los ampliamente estudiados temas de la gestión del cambio, la psicología organizacional y la resistencia al cambio dentro de las organizaciones (Robbins y Coulter, 1996; Kotter, 2001; Duck, 2001; Sabattini y Crosby, 2008).

Actualmente, la transición a sistemas flexibles en el ámbito empresarial es muy rápida, sobre todo en comparación con el sector público. Los modelos de gestión que emplean sistemas flexibles han demostrado su eficacia para alinear los procesos a la estrategia y reflejar las medidas de desempeño en las áreas orgánicas, de gestores y de operarios, en definitiva de toda la firma como un sistema con información, límites, procesos y relaciones (Arrow, 1974; Tirole, 1994; Kaufman, 2000; Watkins, 2007). Esta evolución es sobre todo visible en el caso de empresas que participan en sectores económicos y mercados con un alto grado de rivalidad
(Kaplan y Norton, 1993; Porter, 1998) en los cuales, de acuerdo con Chandler (1977), la mano visible de la gerencia es un factor clave para el predominio de los grandes negocios en diversas industrias.

En esta transición, la producción intelectual asociada a la evaluación del desempeño en el nivel de la firma ha sido generosa, como evidencia la vasta literatura sobre el tema (Kaplan y Norton, 1996 y 2001; Oakland, 2004) y el extenso inventario de marcos de referencia, metodologías y herramientas asociado a los sistemas de gestión y control estratégico que comprende, entre otros, la administración por objetivos (APO), los presupuestos, las métricas financiero-contables, el costeo basado en actividades, los modelos de gestión de la calidad (como el Malcolm Baldrige) y los cuadros de mando integral (como el Balanced Scorecard) y, más recientemente, las herramientas de valor económico agregado (EVA) y gerencia basada en valor (GBV).

En el campo de la gestión pública o gubernamental, la producción de, y la transición hacia, sistemas y tecnologías de gestión que permitan evaluar la eficacia de las estrategias, la productividad del proceso de gestión y las medidas de desempeño en varios niveles de la organización no han seguido la misma trayectoria. Esta afirmación encuentra un sustento abrumador tanto desde la academia como desde la praxis. Investigaciones de autores como Barzelay (2000), Longo (2000) y Joyce (2001) dan cuenta de un creciente número de gerentes en el ámbito de la gestión pública que no disponen de fuentes que les sirvan de guía para trabajar sobre los problemas cotidianos de la gestión de servicios públicos; así como de la necesidad de desarrollar enfoques conceptuales y metodológicos para atender los requerimientos de la nueva gestión pública.

Es de fundamental importancia notar que los modelos de gestión prevalecientes en el ámbito gubernamental aún se mantienen bajo la influencia del enfoque de "organización y métodos», que tuvo su máximo auge en la primera mitad del siglo XX, como parte de las rutas de pensamiento asociadas a la administración científica (Taylor, 1911) y los modelos mecanicistas y de la burocracia racional (Weber, 1947). La característica esencial de estos modelos es el predominio de la estructura como eje de la gestión. Por 
un lado, la dimensión de la estructura comprende los modos de organización y trabajo en función de reglamentos, la discrecionalidad restringida de los gestores, las operaciones y los procesos en función de procedimientos poco flexibles, un diseño organizacional de tipo jerárquico-funcional, y el ejercicio del poder y la toma de decisiones concentrados en la parte más alta de la jerarquía organizacional (Mintzberg, 1989). Por otro lado, la alta estructuración de la gestión implica un fuerte grado de control en los procesos, los procedimientos y el empleo de recursos, los cuales, al ser públicos, justifican la supremacía de modelos rígidos de gestión.

En el caso del Perú, también se debe considerar los esfuerzos y los compromisos de los gobiernos de la última década por impulsar la descentralización del país y la modernización del Estado; con especial énfasis en transitar desde una gestión por actividades a una gestión que produzca resultados. Estas declaraciones políticas han sido particularmente más intensas desde que el Perú iniciara un proceso de descentralización y que los nuevos gobiernos subnacionales asumiesen competencias para promover el desarrollo y empezasen a ejercer, aunque de modo lento, grados crecientes de autonomía administrativa, financiera y de gestión.

Además, las observaciones de los autores sobre el ámbito específico de los gobiernos locales en el país han comprobado que los sistemas de gestión imperantes se mantienen dentro de enfoques mecanicistas y de burocracia racional, cuyas características, bajo el original enfoque de Weber (1947), confieren a las relaciones de poder y autoridad, y a los cuerpos de normas y procedimientos, el papel central en la gestión y la eficiencia técnica de la organización; de lo que se desprende, para efectos de este estudio, que son los presupuestos y los ratios contables la medida eficiente del desempeño. Con esta óptica, Gouldner (1964) y March y Simon (1993) hallan discrepancias entre los postulados de la escuela estructuralista weberiana (que se basa en la racionalidad y la formalidad) y el propósito de lograr mejores niveles de desempeño en organizaciones mecanicistas. De particular importancia resultan las relaciones de poder y el desempeño de las organizaciones (Clegg, Courpasson y Phillips, 2006), así como las fuerzas que se oponen a los límites para la coordinación interdepartamental, la concentración del poder y las decisiones en la parte superior de la pirámide organizacional y los efectos disfuncionales de una supervisión muy estrecha cuando el alcance del control es muy grande o cuando surgen circunstancias que no han sido consideradas por los reglamentos.

En suma, los desarrollos en la teoría de las organizaciones han demostrado que la gestión de carácter operativo y basada en las normas y los procedimientos es subsidiaria de la gestión de carácter estratégico y holístico; lo que coincide con las descripciones de autores como Metcalfe y Richards (1987) y Kenny (2005), quienes procuran marcar las diferencias entre las dimensiones operativa, asociada a operaciones y actividades, y estratégica, eje de los sistemas de control estratégico de gestión de las organizaciones.

Un balance sobre los enfoques adoptados en el proceso de gestión en el ámbito empresarial y el ámbito gubernamental nos remite, por tanto, al clásico debate entre estructura y estrategia, el que cobró realce a la luz de los aportes en la rama de la gerencia estratégica que ha tenido a Chandler (1977) y Mintzberg, Ahlstrand y Lampel (1998) entre un nutrido grupo de académicos para quienes el proceso estratégico es iterativo y sigue una lógica incremental (muchas veces intuitiva), toda vez que las organizaciones realizan cambios significativos en su estrategia y este cambio afecta a la organización y las decisiones a su interior. Lo que resulta decisivo sobre la estructura, el tipo de organización y el modo de trabajo.

Acerca del proceso estratégico, en la escuela prescriptiva destacan Chandler (1962) y Mintzberg (1989), quienes enfatizaron el lema: «La estructura sigue a la estrategia», pues dan por sentado que una estructura es rediseñada para llevar a cabo una nueva estrategia. Por otro lado, en la escuela descriptiva se fundamenta que la estrategia debe ser posterior a la estructura y amoldarse a esta última. Esta línea de pensamiento ha sido particularmente fértil en sus aportes en años recientes, particularmente en el ámbito empresarial, donde el proceso estratégico requiere considerar tanto variables incontrolables del entorno (como las características propias de cada sector económico, el clima de negocios, la institucionalidad y la estabilidad jurídica 
y las reglas de juego en los mercados), como variables internas más sutiles (como las características culturales, el comportamiento organizacional y las preferencias de los grupos de interés dentro de la organización, por ejemplo, los sindicatos). En todo caso, según estos autores, las organizaciones en el ámbito público aún no están influidas por la multiplicidad de realidades y contextos de la estructura existente que las conduzcan a un conflicto entre estructura y estrategia que lleve a privilegiar la primera sobre la segunda.

Se debe señalar que cualquiera que fuese la corriente de pensamiento adoptada (prescriptiva o descriptiva), las dimensiones de ambas, estructura y estrategia, son indisolubles e interdependientes; tal como lo advirtieran Lawrence y Lorsch (1967) en su investigación sobre los problemas de la diferenciación y la integración, y Galbraith (1973), quien profundizó en las dificultades de la organización altamente estructurada para enfrentar eventos no planificados y la necesidad de acciones coordinadas. De modo que no es posible hablar de una organización eficaz que solo privilegie la estrategia o la estructura. Lo que significa que no se puede aplicar sistemas modernos de control estratégico y medición del desempeño en organizaciones donde impera la estructura; ni es posible emplear sistemas de control estratégico rígidos en organizaciones que se conducen en forma estratégica. Esto implica que, a pesar de lo mucho que se ha escrito sobre la aplicación de enfoques de la gestión de empresas al ámbito gubernamental, la escasez de evidencia a favor alerta sobre lo incipiente de la producción de tecnologías de gestión que sirvan para transformar con éxito los modelos de gestión muy estructurados en modelos orientados hacia la eficiencia y la producción de resultados, en virtud de lograr visibilizar el desempeño organizacional como efecto del proceso iterativo entre estrategia y estructura.

Al adoptar un enfoque conciliador entre estructura y estrategia, este artículo va más allá de la toma de conciencia y presenta una herramienta para visibilizar el desempeño de una organización gubernamental mediante la ubicación de la gestión de procesos como la bisagra entre ambas dimensiones (Chenhall, 2005). La herramienta, denominada «curva de desempeñoestructura», emplea ambos ejes para medir la producti- vidad de la organización y, por ende, su capacidad para producir resultados. La estructura refleja el grado en que los procedimientos y la organización del trabajo (como parte de la burocracia) facilitan o dificultan el flujo de los procesos en marcha para la obtención de resultados, mientras que el desempeño mide el grado en que los procesos responden a la estrategia que la organización selecciona para aproximarse a los resultados esperados.

Con el fin de visibilizar el aporte de esta herramienta en la gestión de organizaciones en el ámbito de los gobiernos locales, en este artículo se desarrolla un primer paso que es fundamental para abordar el control estratégico y la evaluación del rendimiento como medidas de la productividad de la organización. Este consiste en dos elementos: el primero comprende expresar la gestión de procesos como la bisagra entre las dimensiones de la estructura y la estrategia; y el segundo implica expresar bajo un lenguaje de indicadores estratégicos y tácticos (u operativos) el grado en el cual los procesos (dimensión estratégica de la gestión) y la organización del trabajo (dimensión operativa de la gestión) requieren estar alineados a las estrategias de, y a los resultados esperados por, la organización.

Este artículo está organizado en cinco secciones. La primera aborda las particularidades del desempeño en el nivel de las organizaciones (con énfasis en el ámbito gubernamental) y la comprensión de los resultados esperados como medida agregada de la productividad y/o valor generado por las organizaciones, a través de uno o más procesos que configuran el modelo de gestión. Estos aspectos permiten, en la segunda sección, describir los fundamentos de la curva de productividad desempeño-estructura de una organización gubernamental y entender ambos aspectos en función de una estrategia en permanente evolución, tal como lo refieren las teorías administrativas establecidas. Finalmente, en las tres últimas secciones se expone la secuencia metodológica para transformar el modelo de gestión gubernamental desde una lógica funcional rígida, basada en actividades y procedimientos, hacia una lógica de procesos, basada en el control de la estrategia y la evaluación de los resultados por medio de indicadores. 


\section{EL DESEMPEÑO ORGANIZACIONAL: MEDIDA DE PRODUCTIVIDAD Y COMPETITIVIDAD EN LA ORGANIZACIÓN}

El empleo de medidas de desempeño de los individuos tiene una larga historia. A principios del siglo pasado eran comunes las métricas de desempeño basadas en el cálculo de tiempos y movimientos y su comparación con medidas estandarizas, cuyos valores obedecían a un flujo de procedimientos dentro de un proceso predeterminado o modelo. El ambiente de negocios, el clima organizacional interno y la competencia, fundamentalmente, alentaron a teóricos y gestores a indagar sobre medidas de desempeño que fueran más allá de lo estático de las medidas convencionales. Las nuevas métricas de desempeño fueron progresivamente evolucionando hacia sistemas enteramente articulados tanto a los resultados operativos como a las metas de nivel estratégico que proyectaran una imagen aproximada del desempeño futuro de la organización.

Con igual razonamiento aumentó el interés por medir la contribución de los actores individuales (áreas y personal) al valor generado por la organización. Primero a través de ratios contables y financieros y métricas, pasando por el empleo de presupuestos y el costeo basado en actividades, hasta indicadores que miden el valor agregado asociado a la labor gerencial y la eficiencia operativa. Esta progresión conjunta condujo a complementar los sistemas de gestión con criterios de evaluación del desempeño, bajo la noción que actualmente se conoce como «control estratégico y medición del desempeño».

Esta evolución, lejos de haberse estabilizado, ha continuado a un ritmo acelerado; tanto en el surgimiento en nuevas aproximaciones teórico-prácticas como en su difusión entre las organizaciones más dinámicas. Los supuestos sobre que el desempeño de los individuos y las personas al interior de la organización es un insumo clave para medir el desempeño de la organización ya han sido confirmados, pero también se ha confirmado la influencia descendente que tienen las políticas de mayor nivel y la labor gerencial sobre el desempeño y el logro de metas de las áreas operativas y el personal que labora en esas áreas.
La evolución desde lo que se ha denominado estrategia y estructura conduce, por tanto, a ratificar que la gestión es un gran proceso de transformación de información, insumos, roles interdependientes y propósitos que atraviesa la organización, cuya marcha armónica o ciclo depende estrictamente de las interacciones entre sus componentes (procesos específicos) y, particularmente, de lo acertado que resulte su proceso estratégico. El objetivo de toda esta lógica es maximizar la eficiencia y la eficacia de las decisiones y las acciones que realiza una organización; lo que puede conducir, por ejemplo, a simplificar o estandarizar acciones, procedimientos y operaciones rutinarios o estructurados, aquellos donde un mismo tipo de decisiones, acciones y conductas predefinidos tienen el mismo o muy similar efecto, o a crear condiciones para que los gestores consigan una respuesta efectiva ante situaciones que no han sido previstas o no han sido consideradas en las políticas o los reglamentos, lo que cae dentro de las nociones de discrecionalidad de los gestores, la teoría de agencia de Coase (1937) y la flexibilidad o la adaptabilidad de la organización ante su entorno.

Dentro de este razonamiento existen configuraciones «tipo de organización-tipo de entorno» que resultan más eficientes que otras. En el caso particular estudiado, las organizaciones del sector público poseen una configuración que alude al «modelo burocrático», en el cual la toma de decisiones (dimensión estratégica de la gestión) y su implementación (dimensión operativa de la gestión) son eficientes y eficaces en el logro de resultados esperados y en un contexto o un entorno de baja complejidad; es decir, la gestión en torno a relaciones de poder y autoridad, reglamentos y procedimientos puede ser muy eficaz y eficiente (alta productividad y alto desempeño) si la complejidad de los procesos que se administran no excede un nivel crítico. La excepción ocurre en el caso de decisiones subóptimas que son bien implementadas $y$, por tanto, no brindan los resultados deseados o esperados.

No obstante, una lectura dinámica evidencia que es difícil aceptar el supuesto de que la complejidad en el entorno o el contexto no va a variar. En contextos de alta complejidad, la configuración tipo de organización-tipo de entorno va a determinar que la estructura no pueda llegar a un nivel crítico de eficiencia, lo que 
se reflejará en baja productividad y pobre desempeño, y esto es precisamente lo que sucede en el ámbito de la gestión gubernamental local, en el que ha ocurrido un aumento de la complejidad, en términos simples, del conjunto de funciones, roles y procesos a cargo de las administraciones gubernamentales.

De modo general, y considerando el supuesto de que en el macroproceso de gestión se contemplan los aspectos de diseño organizacional (estructura y estrategia), roles organizacionales (niveles jerárquicos y relaciones de poder y autoridad), información (entorno y bondad de la estrategia) y aspectos eminentemente técnicos (alineación de los procesos a las estrategias y los resultados esperados), la única forma en que la organización (pública o privada) tenga un desempeño crítico eficiente (alta productividad) radica en adoptar y administrar todo el proceso de gestión bajo una lógica incremental y de procesos (gráfico 1) para poder controlar y retroalimentar el sistema de decisiones y la marcha de la estrategia de la organización, tanto a través de reflejar los resultados de la organización ante su entorno, como de ponderar la organización interna del trabajo. En síntesis, el desempeño de la organización será función directa de la gestión de la estructura y la estrategia.

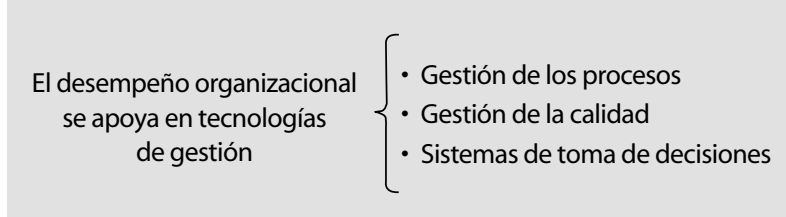

Gráfico 1

Desempeño organizacional en función de procesos y decisiones óptimos

El caso de entidades gubernamentales como las municipalidades es particularmente interesante. En el contexto peruano, la gestión es altamente estructurada (modelo burocrático) y los procesos resultan subsidiarios de los procedimientos, cuando lo racional es que sean los procedimientos los que deben ser subsidiarios de los procesos, como lo ha demostrado la teoría de sistemas. En las entidades municipales persiste el énfasis en controlar la marcha del ámbito interno a través de normas e instrumentos basados en pro- cedimientos administrativos articulados a un cuerpo de normas externas, que por lo general proceden del nivel del gobierno central y de la legislación nacional en materia de funciones orgánicas.

Esta situación impide que las municipalidades logren operar los dos grandes ejes que determinan el éxito en la gestión: el desempeño de la estrategia (productividad) y la flexibilidad de la estructura (burocracia), de modo que una mejora en la marcha de las áreas funcionales y administrativas no necesariamente tiene impacto sobre los resultados de la organización si la marcha de los procesos no ha atravesado también por la mejora correspondiente. De modo inverso, una mejora en la definición y la estandarización de los procesos tampoco tendrá impacto sobre los resultados de la organización si el grado de burocracia y el estado de los procedimientos no han sido racionalizados.

Bajo este razonamiento es posible proponer un esquema que grafica la relación directa e inversa entre desempeño y estructura (o productividad versus burocracia) y que permite apelar a dos variables de la gestión de organizaciones (esquema de dos dimensiones): desempeño en función de la marcha de los procesos y burocracia en función del grado de estructura de sus procesos y procedimientos. Evidentemente, siempre se podrán incorporar otras variables en la medida que los gestores responsables de la organización o los agentes decidan enfrentar la mayor complejidad de las operaciones de la organización con un modelo de gestión más complejo o multidimensional; por ejemplo, la inclusión de variables como «ejecución presupuestal» 0 «medida en la que los líderes formales y directivos de alto nivel jerárquico personifican a título individual el modelo de gestión». El gráfico 2 muestra el esquema bidimensional que correlaciona desempeño (productividad) y estructura (burocracia) y que presenta claramente diversas posiciones o coordenadas que reflejan la posición actual y potencial de una organización con relación al logro de resultados.

En el gráfico se aprecia el desempeño organizacional como una combinación de dos ejes. El eje $X$ corresponde a la estructura reflejada por el grado de burocracia en términos de déficit o exceso de procedimientos. El eje $\mathrm{Y}$ corresponde a la estrategia medida 


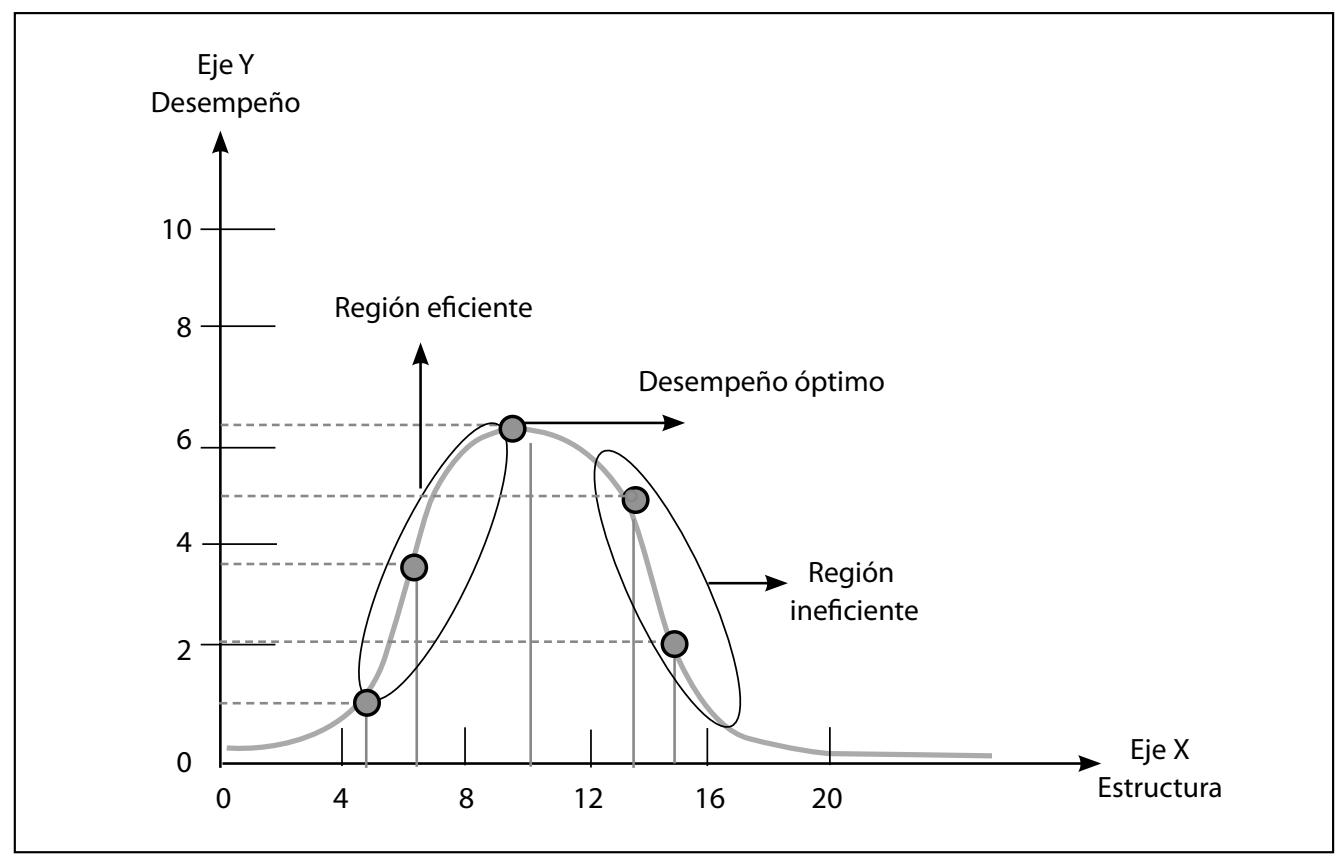

Gráfico 2

Curva desempeño-estructura para estimar el desempeño organizacional

por el desempeño de los procesos en términos de la productividad de estos (eficacia y eficiencia).

En el origen (coordenada 0,0 ) no existen procedimientos (nivel nulo de burocracia y máxima discrecionalidad en toma de decisiones y acciones) y, por tanto, el rendimiento de la organización es nulo o, en el mejor de los casos, incierto. Ascendiendo por ambos ejes, hay además una zona eficiente, formada por numerosas combinaciones (coordenadas) en las que existen cada vez más procedimientos estructurados (por ejemplo, documentos de gestión y normas internas) y una mayor claridad en cuanto a conocer y orientar (gerencia) los procesos de la organización. En esta zona hay ganancias de productividad relacionadas directamente con el incremento de la burocracia.

También se puede identificar un punto de máximo desempeño (coordenadas 10,0;6,2), en el cual el grado de normalización de los procesos y la evolución de la estructura se combinan para otorgar a la organización el máximo rendimiento en términos de productividad de los procesos y los procedimientos o burocracia. Por otro lado, bajo un contexto interno-externo dado, a mayor estructura ya no es posible seguir aumentando la productividad de los procesos; es decir, en coordenadas que van más allá del punto de inflexión ya no es posible seguir aumentando la burocracia para ganar productividad, pues, por el contrario, la productividad disminuye drásticamente y coloca a la organización en la zona de ineficiencia o «pobre desempeño». Este caso es muy frecuente en organizaciones gubernamentales, en las cuales, por ejemplo, se pueden hacer esfuerzos por completar todo el paquete de documentos de gestión, pero no existe evidencia alguna de que el rendimiento de la organización o de los procesos mejore en algo.

En el ámbito municipal, se ha observado que el rendimiento de la organización va de una zona de rendimiento creciente subóptimo (dentro de la zona eficiente) directamente hacia la zona ineficiente o de rendimiento negativo, sin pasar por la zona de máximo desempeño. Esto puede deberse únicamente a que la gestión de los procesos no está articulada a la gestión de la burocracia o al gobierno corporativo de la organización, o a que no existen procesos explícitos bajo control de los gestores; en realidad, los procesos siempre existen pero no siempre se controlan, racionalizan y gestionan. 


\section{LA GESTIÓN Y EL DESEMPEÑO ORGANIZACIONAL BAJO UNA LECTURA DINÁMICA}

El debate entre la estrategia (elección de la organización para tentar una ubicación en su contexto de actividad y planificar resultados en perspectiva) y la estructura (elección de la organización del trabajo que explicita los mecanismos y los roles formales al interior) gira, en resumidas cuentas, en torno a conducir la organización hacia el punto de máximo rendimiento. Esto es posible mediante la combinación óptima de la gestión de procesos y la administración de procedimientos, siempre bajo el supuesto de que la estrategia de la organización implica también decisiones correctas que inciden positivamente en el clima laboral y la salud financiera de esta. Estas precisiones son relevantes dada la confluencia de estrategia y estructura que determina el denominado «modelo de gestión», en el cual el desempeño organizacional equivale al nivel de competitividad de la organización en su conjunto.

No lograr la aproximación al punto de máximo rendimiento implica elevados riesgos y costos en el mediano plazo, pues el desempeño y la estructura no son variables independientes. La transformación de ambos estará determinada, por un lado, por cambios del entorno que obligan a la organización a ajustar su estrategia y los resultados esperados, y, por el otro, por cambios en el interior, muchos de ellos como respuesta a una mayor complejidad, que determinan la modificación de la estructura de la organización.

Eventualmente (en el mediano plazo), los procesos y/o los procedimientos podrían no evolucionar en la medida en que lo hacen el entorno y el interior de la organización. En esta situación, la configuración de la curva desempeño-estructura ya no refleja el punto de rendimiento óptimo que está asociado al buen desempeño organizacional, es decir, a su nivel de competitividad.Si este es el caso, sucesivas mejoras en los procesos y los procedimientos aportan solo mejoras marginales dentro de una misma configuración de desempeñoestructura, sin que esto garantice que la organización logre el rendimiento esperado (los resultados).

Si la organización se encuentra a sí misma atrapada dentro de una curva de productividad (ver gráfico 3), la única manera de resolver este dilema es mediante la innovación del modelo de gestión a través de la introducción de mejoras en los procesos y la aplicación de

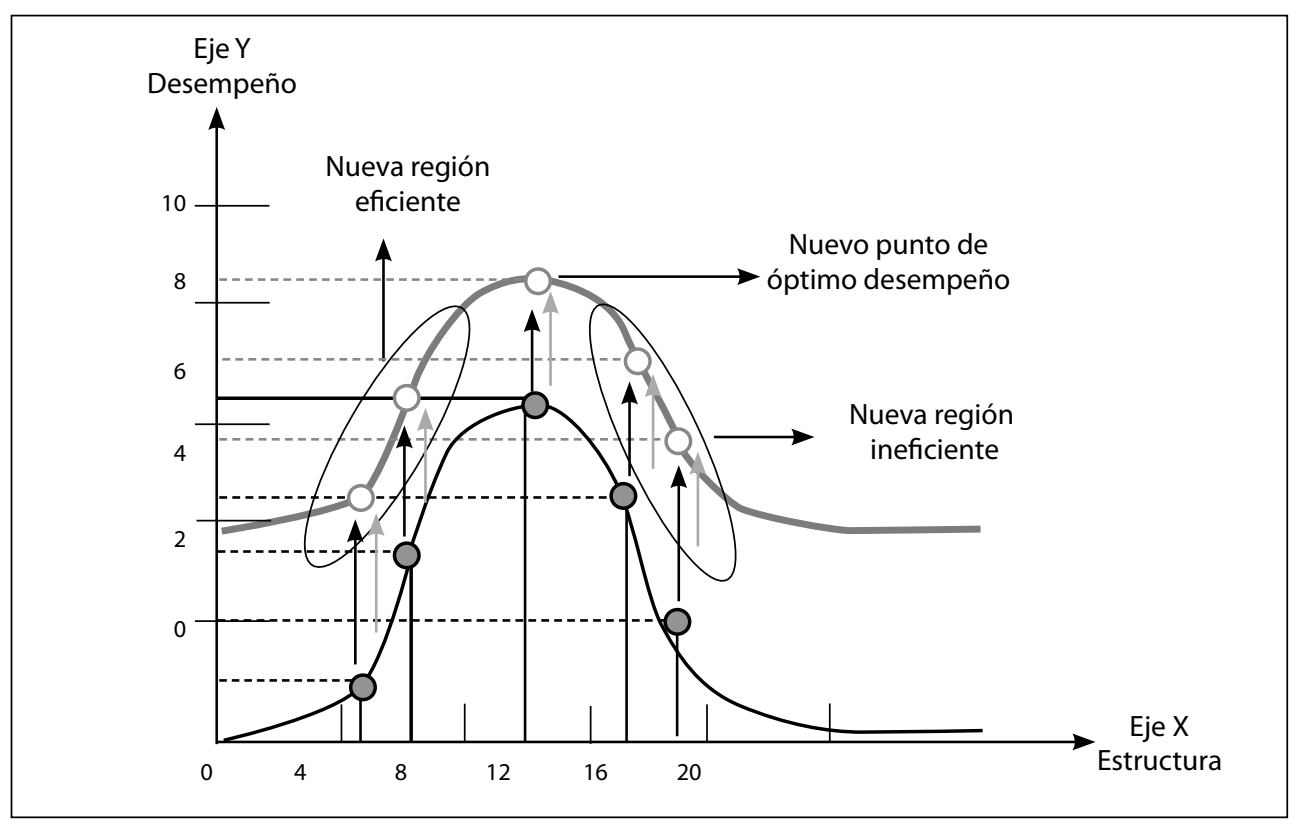

Gráfico 3

La paradoja de la productividad 
nuevas tecnologías de gestión e información, lo que se ha denominado gobierno electrónico y reingeniería de procesos, aplicadas al ámbito gubernamental.

En el gráfico 3, la organización siempre puede decidir su modo de influir sobre su entorno e, incluso, puede reinventar el modo en el cual se organiza internamente, aun en la eventualidad de que la complejidad de las normas o la legislación plantee diversas restricciones a la discrecionalidad de los tomadores de decisiones.

La adopción de tecnologías de gestión (como la reingeniería de procesos o la gestión de indicadores de procesos) y de información (como el gobierno electrónico y los sistemas de soporte a la toma de decisiones) permite a una organización superar la paradoja de la productividad al posibilitar el acceso a una nueva configuración desempeño-estructura, es decir, a una nueva curva de productividad en la cual existe un nuevo punto de óptimo rendimiento y, por tanto, holgura para que la organización continúe aumentando su nivel de burocracia (estructura) en respuesta a un escenario de mayor complejidad, al mismo tiempo que se incrementa la productividad (el desempeño de los procesos).

En conclusión, eventualmente la estructura y el grado de normalización de sus procedimientos terminan siendo obsoletos para lograr los resultados deseados, toda vez que el entorno al que sirve la organización es dinámico y se requieren ajustes en la estrategia, la organización y, en definitiva, el modelo de gestión.

Una intervención aislada, como en el caso de un programa de modernización integral, no dará todos los resultados esperados si solo se trabaja en función de automatizar los procedimientos (hacer un mapa de los procesos actuales y estructurarlos en una nueva configuración), ni con la adopción de una gestión orientada a resultados que implique manejar indicadores de gestión, ni con una redefinición de los roles y las responsabilidades de las áreas, las personas y los tomadores de decisión dentro de la organización. Los resultados en la zona eficiente solo ocurren si todas las variables son trabajadas en conjunto y de modo interactivo, es decir, entendiendo la relación de causalidad (causa-efecto) de una intervención específica en el conjunto de las demás intervenciones que son parte del programa de modernización.

\section{EL PROCESO DE GESTIÓN Y LOS INDICADORES ESTRATÉGICOS Y TÁCTICOS}

Al ser la gestión de los procesos (dimensión estratégica) y la administración de la estructura (dimensión operativa) dos componentes estructurales de todo modelo de gestión en cualquier organización, resulta esencial que los gestores dispongan de herramientas para compatibilizar ambas dimensiones: la estrategia (conducción de procesos) y la estructura (control de operaciones).

En el actual estadio de evolución de la adopción de sistemas y/o modelos de gestión, el empleo de indicadores parece ser el tipo de buena práctica de mayor y más rápida difusión en los campos gubernamental, empresarial y social, particularmente los denominados «tableros de comando». No obstante, en algunos tipos de organizaciones, sobre todo las entidades gubernamentales, las agencias de cooperación técnica internacional y los organismos no gubernamentales (ONG), existe una notoria confusión con relación a qué implican los indicadores y su manejo.

El énfasis en administrar en función de actividades (reducto de los modelos de gestión predominantes durante la primera mitad del siglo pasado) y no de resultados (evolución del pionero modelo de «administración por objetivos» desarrollado en la década de 1980) conduce a maximizar el número de indicadores de gestión e incrementar de modo desmedido la cantidad de datos (no necesariamente su calidad) para monitorear y evaluar un determinado proceso o actividad. Más aun, no son pocos los casos en los cuales, por ejemplo, se otorga a los indicadores sobre el monitoreo de la frecuencia de regadío de parques públicos igual jerarquía que a aquellos que evalúan la eficacia de un programa de planificación familiar dirigido a madres y padres adolescentes.

Las manifestaciones de esta confusión en torno a los indicadores de gestión son numerosas y muy diversas. Muchas de ellas radican en la falta de información 
y cabal comprensión acerca de las dimensiones de la gestión en las organizaciones. Este es el caso de no caer en la cuenta de que, además de la dimensión humana, la dirección de organizaciones también comprende las dimensiones de toma de decisiones (modelo político y modelo racional), gestión de procesos y grado de normalización de las funciones, las actividades y los procedimientos. Esto nos lleva a entender que la gestión tiene dos dimensiones mutuamente dependientes: la gestión de nivel estratégico, cuyas herramientas se sustentan en el empleo de indicadores estratégicos, y la gestión de nivel operativo, cuyas aplicaciones se sustentan en la construcción y el uso de indicadores operativos o tácticos.

Partiendo del supuesto de que una determinada organización municipal tiene definido cierto proceso (con sus respectivos procedimientos) y que este es transversal y demanda del concurso de distintas áreas programáticas, de soporte administrativo y apoyo, el gráfico 4 muestra un conjunto de 6 indicadores de nivel estratégico que, a su vez, constituyen los puntos críticos de control del proceso.

Es oportuno señalar que los indicadores estratégicos son los que específicamente reportan resultados o el estado actual en el logro de una meta. Los indicadores tácticos u operativos son los que reportan sí las actividades programadas (el evento) están siendo ejecutadas y el valor que «el evento» toma en un determinado punto de control. Como fácilmente se puede comprender, ambos tipos de indicadores son imprescindibles para la gestión; más aun, si bien los indicadores estratégicos reflejan la medida del desempeño o el logro, deben construirse a partir de indicadores operativos, los que no necesariamente han de ser recolectados y procesados por una misma área de la organización.

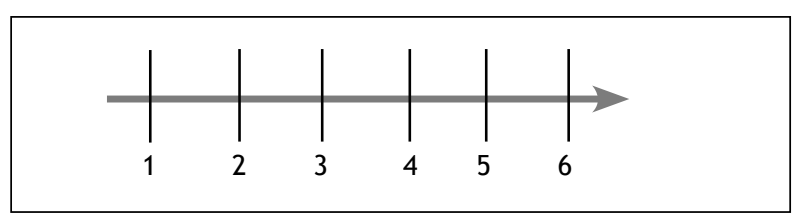

Gráfico 4

Indicadores dentro del proceso de recolección de residuos sólidos domésticos
Donde:

Indicador 1: disponibilidad de presupuesto debidamente sustentado en el flujo de caja asociado a las partidas contables de recursos corrientes de la municipalidad.

Indicador 2: meta de cobertura del servicio en función de la demanda actual y proyectada.

Indicador 3: programación de rutas actuales y proyectadas en función de los datos de los indicadores 1 y 2 .

Indicador 4: nivel de operatividad, vigencia tecnológica y mantenimiento de los equipos y los vehículos.

Indicador 5: nivel de capacidades y eficiencia operativa del personal operativo y administrativo.

Indicador 6: medidas que reflejan la capacidad libre del relleno sanitario, considerando la homogeneidad en la distribución de las zonas de deposición y la discriminación por tipo de residuo.

Evidentemente, todos los indicadores señalados son indicadores operativos con cierto grado de complejidad. Ninguno por sí solo refleja el resultado esperado, el cual podría definirse como: «La evolución de la capacidad y la escalabilidad del proceso de recojo y deposición selectiva de los residuos sólidos domésticos en un determinado periodo o ciclo».

En función de la definición explícita del «resultado esperado», los participantes del proceso, sobre todo el área gestora y coordinadora, se verían en la necesidad de desarrollar el algoritmo o la fórmula para expresar el indicador estratégico que brinde una idea bastante aproximada del resultado, el cual en este caso es una medida de satisfacción de la demanda actual y de flexibilidad con relación a la demanda proyectada y la capacidad, el equipamiento y la suficiencia presupuestal de la municipalidad en ese proceso en concreto.

No obstante, aún son poco frecuentes los casos en los cuales los gestores de este tipo de procesos disponen de las condiciones, la motivación o, en una situación extrema, la preparación para ir más allá del reporte de medidas operativas y trabajar en función de indicadores estratégicos críticos que, además, reflejen la articulación al interior de la municipalidad con otras áreas de programación, administración y apoyo.

Otro de los aspectos que refleja el empleo poco eficaz de los indicadores estratégicos y tácticos es 
el relativo al número de indicadores. Intuitivamente se puede afirmar que disponer de más indicadores no implica necesariamente llevar un mejor control sobre la efectividad de la gestión, las decisiones y el desempeño organizacional. Al tener el gestor exceso de información le será más difícil discriminar cuál es la que realmente necesita y, como respuesta, priorizará la toma intuitiva de decisiones o su propio mapa mental. No obstante, en el ámbito particular de la administración pública aún se puede hallar casos en los que se crean numerosos indicadores, se implementan algunos de ellos sin un criterio debidamente justificado $y$, finalmente, no se utiliza ninguno para la gestión concreta. ¿Por qué sucede esto?

En el ejemplo anterior, sobre el proceso de recolección y deposición selectiva de residuos sólidos domésticos (gráfico 4), se identificaron actividades críticas que debían cumplirse para que el proceso fluyese en función de una secuencia también crítica (una hoja de ruta). Esta definición de actividades críticas permite, por un lado, racionalizar el proceso mismo $y$, por el otro, identificar los indicadores tácticos u operativos que, en su momento, servirán para construir los indicadores. Si bien ya se hizo referencia a esta situación, conviene retomarla para responder por qué, en términos generales, existe una inclinación de los gestores a contar con muchos indicadores para eventualmente usar solo algunos — sin que necesariamente sean los más relevantes, puesto que el gestor siempre estará sujeto a sus preferencias y mapas mentales-o ninguno.

En la definición explícita de los indicadores operativos asociados a actividades críticas también se debe entender que distintas áreas requieren trabajar de modo coordinado, si es que el área responsable del proceso necesita operar mediante un modelo de gestión a través del empleo de indicadores. Por ejemplo, los indicadores del 1 al 6 aclaran que el proceso tiene una instancia coordinadora que lo conduce (puede ser la gerencia de Servicios a la Ciudad), concibe, gesta, monitorea y evalúa junto con otras áreas como planificación y presupuesto, desarrollo urbano, administración y finanzas, logística, proyectos especiales, parques $y$ jardines y recursos humanos.
Lo óptimo, el punto de máximo rendimiento (la curva desempeño-estructura), coincide con un estado en el cual las áreas relevantes de un proceso coordinan sus acciones en función del conjunto de indicadores estratégicos y operativos del proceso. No sería difícil verificar que la medida en que aumenta el grado de articulación en torno a un proceso relevante entre los distintos departamentos es inversamente proporcional al número y la importancia de los indicadores operativos $y$, por ende, estratégicos. La articulación al interior actúa como un mecanismo que restringe el exceso de complejidad y burocracia alrededor del número de procesos y la extensión del proceso, medida en términos del número de procedimientos y puntos de control asociados a la regulación gubernamental y la reglamentación interna.

En correspondencia, un bajo grado de articulación interna corresponde a un estado en el que cada área genera y administra procesos y procedimientos en exceso; lo que da origen a un número cada vez mayor de indicadores que no necesariamente «conversan» con los indicadores que generan y administran las demás áreas de programación, administración y apoyo. Esto no implica que exista un único número eficiente de procesos (con sus respectivos indicadores estratégicos) y de procedimientos $u$ operaciones (con sus correspondientes indicadores operativos) para un mismo tipo de institución gubernamental.

El tamaño de la organización es fundamental y equivale a la estructura orgánica como respuesta a la complejidad del entorno. Así, por ejemplo, un proceso en el ámbito del desarrollo económico local de nivel provincial demanda tipos de intervención de mayor alcance, complejidad y magnitud de recursos que lo que requerirá una municipalidad distrital. El número de áreas internas y actores en el entorno con los que hay que trabajar de manera articulada es mayor en la municipalidad provincial, lo que determina procesos más complejos, con indicadores estratégicos más finos, y más procedimientos, con una automatización de la estructura y la burocracia (ver gráfico 5).

En el gráfico 5 se aprecia un proceso extenso de gestión que demanda el manejo de 9 indicadores 


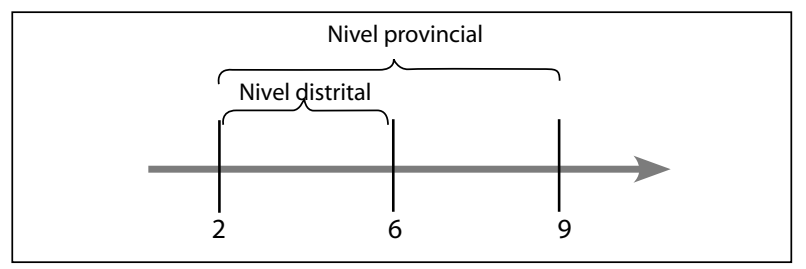

Gráfico 5

Escala de comparación de la extensión de los procesos provinciales $y$ distritales

estratégicos para el nivel provincial, mientras que solo requiere 6 indicadores estratégicos para el nivel distrital. En ambos casos es posible que solo se necesiten 2 indicadores para que el proceso exista; pero en este escenario será sencillo verificar que la insuficiente estructura (nivel subóptimo de burocracia) ocasiona dos efectos que reducen el desempeño organizacional: a) no existen condiciones para una efectiva articulación entre los departamentos que contribuya a los resultados esperados del proceso y b) el trabajo aislado provoca que las áreas compensen la desarticulación a través del aumento de los procedimientos y las operaciones necesarios para alimentar el proceso (exceso de burocracia), lo que significa que la organización pasa directamente de la zona de poca eficiencia a la zona de ineficiencia, sin atravesar el área de óptimo rendimiento dentro de la curva «desempeño-estructura».

En conclusión, en la medida en que la definición de los procesos sea deficiente, la desarticulación interdepartamental sea incipiente y la compensación de ambos aspectos previos a través del aumento de los procedimientos y la regulación interna (que consume tiempo) genere un exceso de burocracia, la propensión hacia emplear muchos indicadores operativos poco relevantes (aquellos que no contribuyen con la construcción de los indicadores estratégicos o «por resultados») será mayor y, eventualmente, los tomadores de decisiones o los gestores se verán incentivados a dejar de emplearlos ${ }^{3}$.

3. Aunque la conclusión planteada se sustenta en modernas teorías administrativas y microeconómicas, y en la experiencia de campo, aún faltan estudios que profundicen en la validación de estas afirmaciones y sus implicancias y revelen otras consecuencias para el funcionamiento de la organización.

\section{AJUSTE DE PROCESOS EN FUNCIÓN DE LA RACIONALIZACIÓN DE LOS INDICADORES}

La sección anterior trató sobre la definición de indicadores como una función de las características del proceso, los resultados esperados y la estrategia de la organización en su gestión tanto interior como hacia el entorno. Se postuló la relación inversamente proporcional entre el grado de articulación entre las áreas que participan en un proceso y el número de indicadores, enfatizándose que un diseño eficiente de indicadores estratégicos requería seleccionar los indicadores operativos más relevantes. El número de estos está también determinado por los puntos de control, es decir, los momentos en que es necesario verificar si el evento (el proceso) ocurre y qué valores toma el indicador (la medida que refleja el indicador) en cada punto.

En esta sección se expondrá una metodología rápida para definir los indicadores operativos y los criterios que orientan la determinación del número eficiente de indicadores estratégicos y su definición explícita, es decir, su construcción y normalización. Con este propósito, el gráfico 6 muestra un proceso en el que se han identificado 20 puntos de control que reflejan la necesidad de definir (denominar y explicar) por lo menos 20 indicadores tácticos $\mathrm{u}$ operativos. No obstante, el mapeo del proceso, el análisis del grado de articulación interdepartamental y la reflexión profunda sobre los resultados esperados del proceso en función de objetivos o metas de logro en el nivel institucional (en el área específica que da origen al proceso) alertarían sobre el riesgo de implementar una gestión por procesos (empleo de indicadores que reflejen el logro de resultados) en el estado actual.

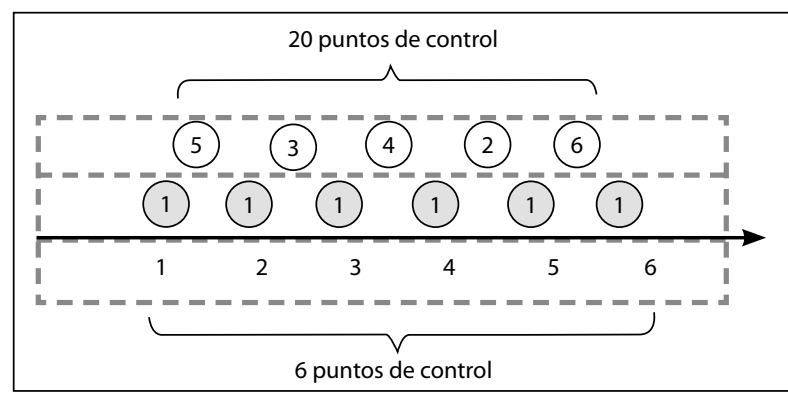

Gráfico 6

Asociación de indicadores operativos en función de los resultados esperados 
Las informaciones derivadas del mapeo del proceso y de los otros aspectos referidos podrían emplearse para emprender una reforma focalizada del proceso y de la estructura y los roles y las responsabilidades, pues una modernización no necesariamente debe ser integral.

La reforma se conoce también como reingeniería de procesos desde una perspectiva doble:a) optimización del flujo transversal del proceso y b) simplificación administrativa. Como parte de la reforma y el rediseño preliminar del nuevo proceso es posible que los puntos de control se reduzcan de 20 a algo más de 6 , lo que implica tomar una primera decisión: «¿Están los 6 puntos de control en un nivel en el cual ya es posible reportar resultados del proceso?».

Si los 6 puntos de control contienen la información que permite tomar decisiones, evaluar resultados y brindar lineamientos para la definición de políticas sobre el funcionamiento de las áreas que intervienen en el proceso, es posible tener una primera aproximación a los indicadores estratégicos. Esta óptima situación es algo esquiva en la práctica. Lo que en el mejor de los casos puede ocurrir es que algunos de los puntos de control estén preparados para el reporte de resultados del proceso, mientras que los puntos de control restantes requerirán de un trabajo de mayor detalle para derivar los indicadores operativos.

No obstante, en el gráfico 6 se ha supuesto que los 6 puntos de control no necesariamente serán aquellos que reporten resultados. Por ello, el siguiente paso consiste en establecer estos puntos como reflejo del tipo de información que debe recolectarse y procesarse previamente en un nivel operativo, lo que dará como resultado la definición de los indicadores operativos. En consecuencia, los pasos posteriores serán:a) derivar una primera aproximación a los indicadores estratégicos relevantes, b) ajustar y desarrollar en detalle los indicadores tácticos, c) ajustar los indicadores estratégicos y d) desarrollar el modelo resultante y efectuar los ajustes necesarios al proceso. Esto es, implementar el proceso y los indicadores y efectuar pruebas para evaluar y comparar resultados sucesivos, mediante varias corridas.

\section{Construcción de indicadores}

Una gestión es, por definición, la administración de procesos y la toma de decisiones (estrategia) y la administración de centros de costos y responsabilidades (estructura) con un propósito definido. En consecuencia, siempre es un proceso que conduce al logro de resultados $y$, a su vez, los resultados son una característica implícita de la gestión. Estrictamente hablando, es una redundancia tratar de discriminar «la gestión» de «la gestión por resultados» (o para resultados), pues «la gestión sin resultados» no tiene sentido en sí misma.

La expresión tangible de una gestión está en el diseño, la implementación, el control y la retroalimentación de los procesos. Es la gestión de los procesos la que en realidad produce los resultados; de modo que una gestión puede «no ser por procesos» (no garantiza resultados) o «ser por procesos», en la cual los resultados se producen aunque no necesariamente en la medida de lo esperado o planificado.

Hacer gestión sin un sustento en el conocimiento y el control sobre los procesos equivale a una gestión basada en la intuición, la experiencia (que no siempre se aplica con buenos resultados cuando cambia el contexto) o restringirse exclusivamente a los procedimientos. Una gestión orientada a los procesos equivale, por tanto, a una gestión estratégica y el manejo de los distintos grados de riesgo que implican las decisiones.

Al combinar la información sobre el análisis del proceso y su articulación a la gestión estratégica (desempeño) y la gestión operativa (estructura) de la organización es posible definir metas de racionalización o reforma del proceso, la funcionalidad orgánica y normativa, y los roles y las responsabilidades. Este primer paso permite una aproximación al nuevo proceso y sus indicadores, especialmente los tácticos.

Esta lógica se puede apreciar en el esquema que se muestra en el gráfico 7, al que a continuación acompañará un ejemplo sencillo que permita aclarar la diferencia entre una gestión que solo se sirve de indicadores operativos y una gestión orientada a resultados, la cual necesariamente utiliza los indicadores de nivel operativo para construir indicadores de nivel estratégico, que 


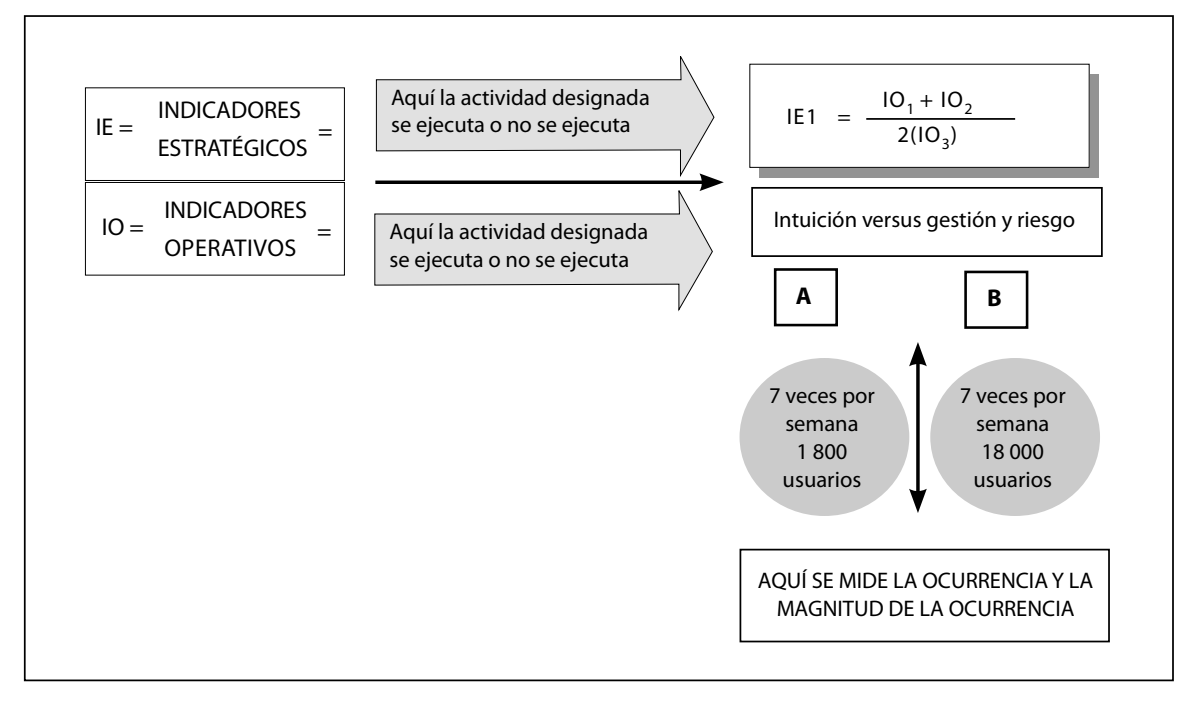

Gráfico 7

Flujo iterativo del diseño de indicadores estratégicos en función de indicadores operativos

son aquellos que permiten medir la productividad del proceso y el desempeño de la organización.

Los indicadores operativos (IO) permiten verificar que un evento bajo control se encuentra en ejecución, es decir, que las actividades y el ciclo del proceso operan bajo los patrones preestablecidos. Asimismo, permiten articular la ejecución de actividades con los centros de costos (enfoque financiero-contable) y/o partidas (enfoque contable) de las distintas áreas que participan en el proceso. Finalmente, permiten medir el estado de la(s) variable(s) que adquiere un determinado punto de control y/o las actividades en un momento dado.

En el gráfico 7 se aprecia un caso en el que se comparan dos situaciones. Ambas se refieren a la acción por la que un camión recolector de residuos sólidos domésticos recorre una ruta predeterminada en dos distritos vecinos ( $\mathrm{A}$ y $\mathrm{B}$ ) con una frecuencia semanal de 7 veces. Si el solo hecho de tomar la variable «frecuencia» como punto de control sirviese para asignar un indicador de resultados, se estaría incurriendo en un grave error al afirmar que el servicio tiene el mismo rendimiento para ambos distritos. Esto se podría comprobar al caer en la cuenta que mientras en el distrito A se atiende a 1800 usuarios, en el distrito B se llega a 18 mil usuarios. El paso lógico sería utilizar ambos datos (frecuencia y demanda) para construir un indicador operativo que refleje el rendimiento del proceso en un punto de control específico, como podría ser la estimación de la cobertura y el cálculo del déficit o la suficiencia del servicio, lo que nos daría un nuevo dato de tipo compuesto.

Los indicadores estratégicos (IE) permiten medir el desempeño de modo sistemático: a) compatibilizan los resultados reales del proceso con lo planificado, b) permiten evaluar el rendimiento de las áreas y los gestores que participan en la ejecución del proceso al vincular el grado de logro de las metas operativas con el rendimiento de las personas, y c) ayudan a evaluar si los procedimientos y la reglamentación interna facilitan u obstaculizan el flujo del proceso. Finalmente, los indicadores estratégicos reflejan la calidad de la toma de decisiones y brindan información para mejorar, ajustar o cambiar la estrategia que justifica el diseño del proceso.

En el gráfico 7 también se puede apreciar que la definición de un indicador de tipo estratégico necesariamente requiere trabajar con datos simples o datos compuestos (indicadores operativos) para explicitar un resultado que permita evaluar el rendimiento de un proceso o una fase en particular dentro del ciclo del proceso. Esta definición de indicadores estratégicos 
debe ser concertada entre los gestores de las áreas que participan en el proceso. Así, por ejemplo, podría determinarse que el indicador estratégico (IE) requiere obtener el cociente entre la suma de los indicadores operativos $\mathrm{IO}_{1}$ e $\mathrm{IO}_{2}$ y dos veces el valor que refleje el indicador operativo $\mathrm{IO}_{3}$. Para generalizar los alcances del razonamiento expuesto en los párrafos anteriores a cualquier tipo de proceso es oportuno recalcar que para justificar un proceso se requieren por lo menos 2 indicadores de nivel estratégico, donde cada uno de ellos puede necesitar reflejar dos puntos de control específicos dentro del ciclo del proceso. Cada punto de control está asociado a dos fases del ciclo del proceso y refleja la información de actividades y procedimientos a través de un determinado número de indicadores operativos.

No obstante, es errado suponer que a cada acción o procedimiento le corresponderá un indicador operativo. Si así fuese se requerirían, por ejemplo, 20 indicadores, lo que constituiría un exceso que eventualmente reduciría la posibilidad de contar con datos relevantes para construir indicadores estratégicos o de resultados. Es ineludible racionalizar el proceso y trabajar con los datos simples para elaborar datos complejos que sí puedan asociarse a indicadores operativos (gráfico 8). Más aun, algunos de los nuevos indicadores operativos podrían, con algunos ajustes, transformarse directamente en indicadores estratégicos. Cada caso es particular.

\section{Ajuste iterativo de indicadores}

La gestión de procesos y el trabajo con indicadores no constituyen solo un ejercicio técnico sino político $y$ técnico. Los procesos y sus indicadores pueden estar bien definidos e implementados e incluso pueden dar resultados auspiciosos, pero ello no garantiza que se esté reflejando el desempeño organizacional. El que la organización (pública, privada o civil) tenga buenos procesos desde el punto de vista netamente técnico no necesariamente significa que cumpla con su misión y logre sus objetivos institucionales.

Por esa razón, se requiere enfatizar que la definición de los procesos se debe exclusivamente a la conducción de toda la organización en función de su entorno

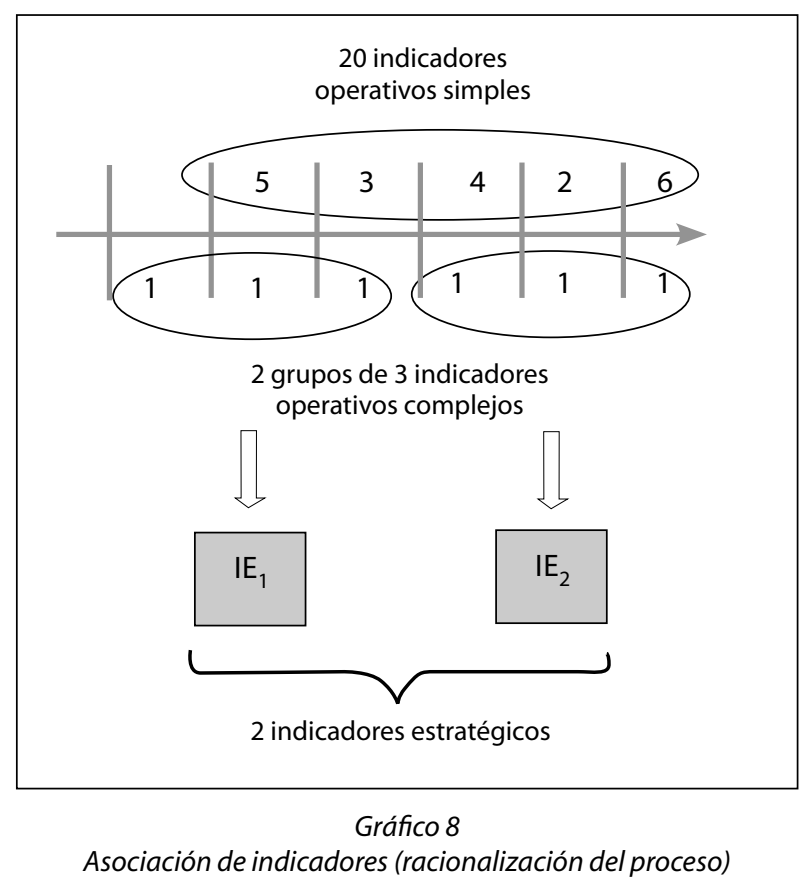

y su visión y misión. Solo mediante esa priorización se puede adoptar y concertar los criterios y las políticas que permitirán expresar qué es lo que se pretende reflejar (en términos de resultados) con cada uno de los indicadores estratégicos. De igual modo, los indicadores estratégicos necesitan estar articulados al día a día, lo que se logra mediante indicadores operativos (gráfico 9).

Es poco prudente aventurarse a reformar procesos e implantar indicadores estratégicos si es que la estrategia de la organización no es clara ni está debidamen-

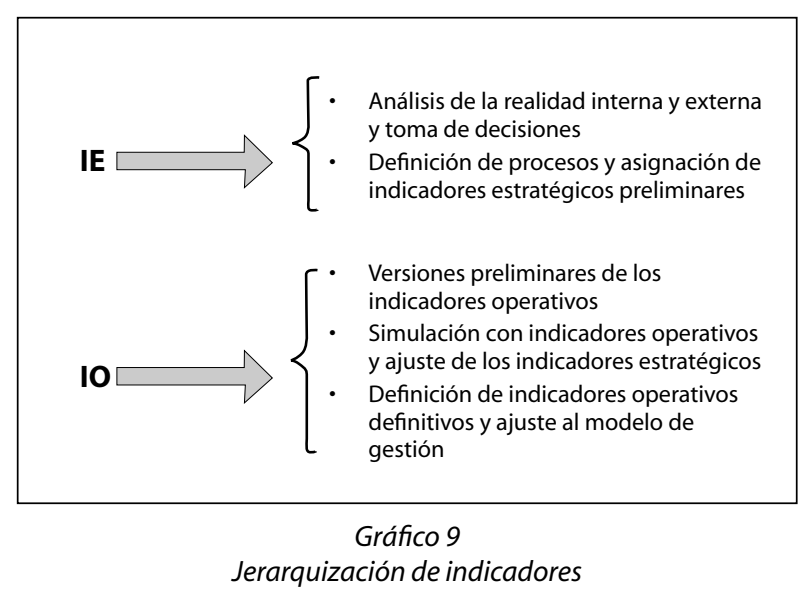


te sustentada mediante un conjunto de informaciones y decisiones de política externa e interna. Esta eventualidad, frecuente en el ámbito gubernamental, no permite que se implementen y gestionen procesos orientados a resultados. Por otro lado, es poco prudente aventurarse a definir indicadores operativos sin tomar en cuenta qué tipo de información aportan para la construcción y la valoración de los indicadores estratégicos. Esta posibilidad, también muy frecuente en todo tipo de organizaciones, no permite que la gestión de procesos contribuya a que la organización obtenga resultados reales.

Incluso si los indicadores operativos se definiesen en función de indicadores estratégicos, no es prudente afirmar que se trata de versiones definitivas. El manejo de indicadores es, en principio, un hecho iterativo (gráfico 10). Durante las pruebas de implementación, las versiones de los indicadores son preliminares, pues es necesario ajustarlos de modo sucesivo mediante simulaciones (pruebas en frío) y ciclos (pruebas en caliente) que permitan adaptar los indicadores, el ciclo, el proceso, la estructura y, en algunos casos, la estrategia de la organización.

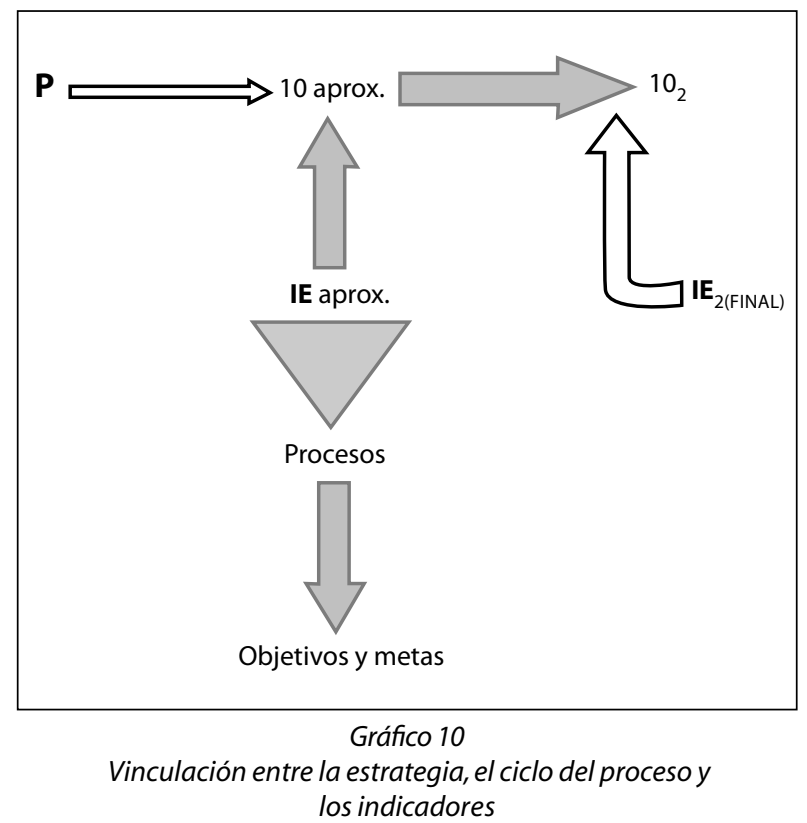

Es importante insistir en que es a partir de la estrategia que se derivan los procesos y el esquema general del MODELO DE GESTIÓN POR PROCESOS. Sobre esta base se obtendrá la primera aproximación a los indicadores operativos y estratégicos; los que, en sucesivas corri-

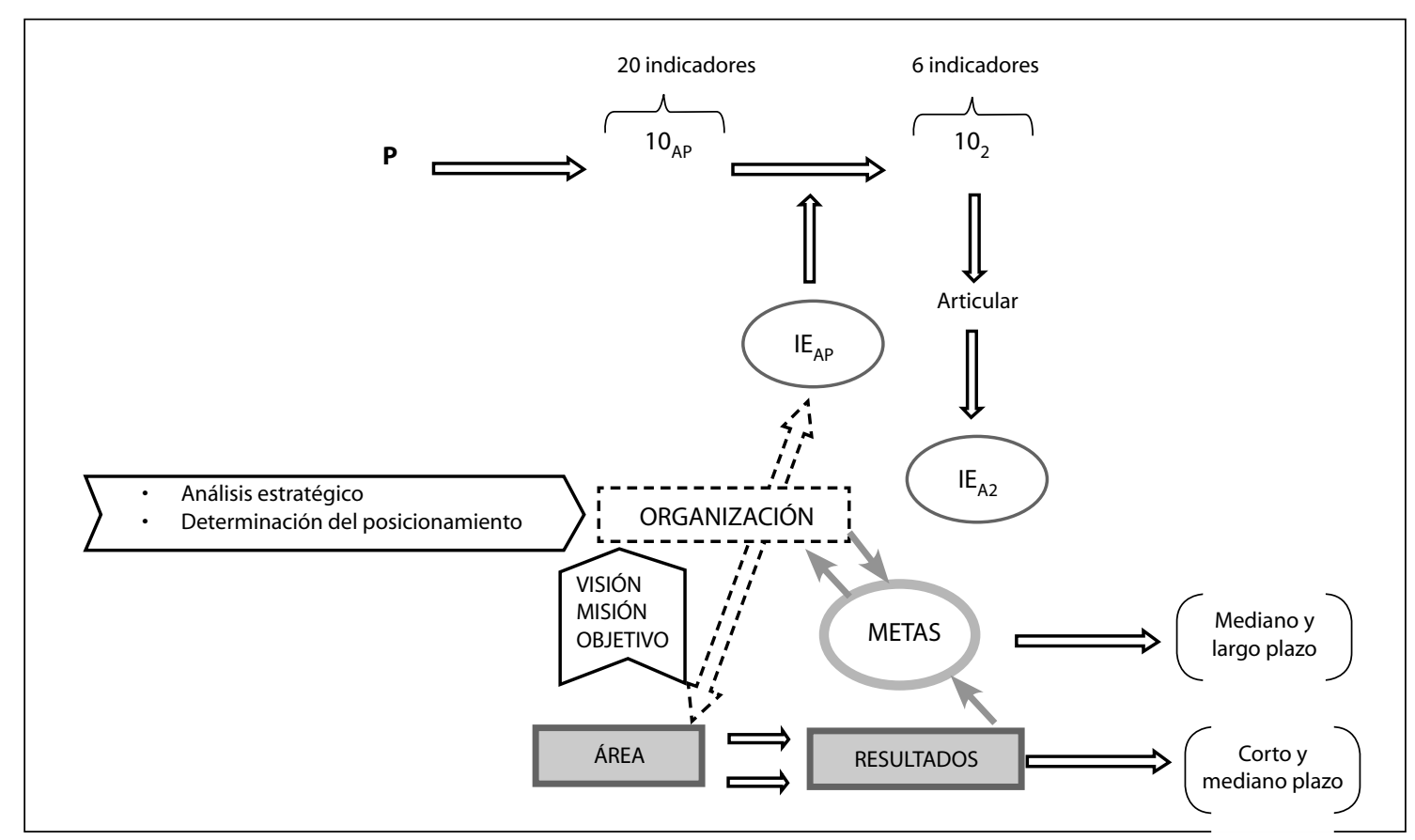

Gráfico 11

Ajuste de indicadores en función de procesos alineados con la estrategia de la organización 
das (pruebas en frío y en caliente), evolucionarán hacia la versión final de los indicadores (gráfico 11), lo que no implica que no se puedan perfeccionar más adelante.

En el gráfico 11 se aprecia el proceso completo de definición y ajuste de indicadores, de acuerdo con los lineamientos descritos. La organización se traza una visión y estrategias que son los insumos para definir los procesos (dimensión de la estrategia) y el arreglo organizacional para operar y dirigir la organización (dimensión de la estructura). Esta información es el primer gran pilar del modelo de gestión y permite derivar un mecanismo o un sistema de control y evaluación del desempeño organizacional que está compuesto por indicadores.

Evidentemente, el empleo de indicadores para reflejar la medida del rendimiento, el desempeño o la productividad de la organización requiere de datos agregados (los indicadores), pero estos nunca lograrán reemplazar el proceso de toma de decisiones y tampoco garantizarán que quienes deciden sean infalibles. Por esta razón, la adopción, el ajuste progresivo y la institucionalización de un MODELO DE GESTIÓN POR PROCESOS se plantea como el paso siguiente y, a la vez, uno de los mayores desafíos de las organizaciones y los gestores.

\section{EL MODELO AJUSTADO DE GESTIÓN GUBERNAMENTAL BAJO UNA LÓGICA DE PROCESOS}

En las secciones previas se ha abordado la medida del desempeño de la organización como uno de los mayores desafíos de la gerencia moderna. Se partió del antiguo dilema de estrategia versus estructura, que tiene sus orígenes en la década de 1930 y se desarrolla desde la administración en la década de 1970, para tratar ambas dimensiones de la dirección de organizaciones de modo combinado.

Asimismo, se destacó que el modelo de gestión que combine a ambas para alcanzar un punto de máximo rendimiento, reflejado en la curva de productividad y desempeño organizacional, necesariamente se sustenta en la adopción de la gestión de procesos; lo que implica alinear los procesos a la estrategia de la organización y controlar la ejecución de la estrategia y los resultados de los procesos mediante indicadores que permitan a los gestores evaluar los resultados organizacionales $y$, sobre esta base, ajustar el proceso de toma de decisiones y la conducción de las operaciones.

En esta sección se busca completar el mapa de la gestión por procesos como núcleo medular de un modelo de gestión. Para hacerlo, se empezará por señalar cuatro errores recurrentes que limitan la potencia de un planteamiento de modelos de gestión por procesos al igual que el empleo de indicadores.

El primer error, y probablemente el más común en la gestión de todo tipo de organizaciones, es el referido a la confusión entre procesos en control, total o parcial, de una organización y procesos sobre los cuales la organización no tiene control alguno, sino una incidencia, débil o fuerte, directa o indirecta.

La noción de procesos es tan antigua como los primeros conocimientos que las civilizaciones clásicas desarrollaron, particularmente la griega. En los siglos sucesivos, la evolución de las ciencias exactas requirió del desarrollo de la noción de sistemas, a modo de una visión estática y en equilibrio de eventos de la naturaleza o creados por el hombre. En la medida en que en el mundo real los sistemas son dinámicos pues jamás permanecen estáticos, los únicos elementos que podrían permitir al hombre entender la dinámica de los sistemas serían los medios bajo los cuales un sistema puede ser controlado, de modo que fuese posible entender a cabalidad las transformaciones parciales en sus componentes $y$, de ese modo, tener una idea bastante aproximada de la transformación que se opera en todo el sistema y así deducir la relación de causalidad (causa-efecto) que produce resultados nuevos a partir de insumos, en virtud de la sucesión de eventos naturales o provocados.

En síntesis, un proceso es un sistema o parte de un sistema mayor, el cual se caracteriza por la permanente transformación de insumos en productos o resultados, sobre los que se tiene un grado de control relativo y proporcional al número de variables del proceso sobre 
las que existe intervención directa, natural o provocada. Un proceso tiene una temporalidad que regularmente es conocida por quienes participan en su funcionamiento y evolución. Desde ningún punto de vista puede el proceso ser un evento indefinido; por el contrario, se conoce su inicio $y$, aproximadamente, su fin, lo que coloca a los procesos dentro de la categoría de hechos de corto y, a lo más, mediano plazo.

En este aspecto de la temporalidad es donde precisamente radica mucha de la confusión con los procesos; puesto que ante algunas tendencias o algunos eventos de los cuales no existe control por parte de quienes los vigilan se suele pensar que se trata de procesos. Ello lleva a la necesidad de mover el punto temporal de referencia a escalas más o menos definidas cuando se pretende intervenir sobre los procesos de largo plazo; por ejemplo, ciclos de cinco o más años. No son pocos los ejemplos en los que las organizaciones municipales, por ejemplo, refieren sus progresos parciales como parte de un proceso sobre cuyo resultado final no tienen responsabilidad (pues solo apor$\tan )$. Este tipo de declaraciones no es semánticamente inapropiado, pero deja muy claro que quienes formulan una intervención con el objeto de mejorar algún aspecto de la realidad local no tienen un compromiso firme con el resultado o el propósito que sirvió de sustento al «proceso» de formulación de la intervención.

El segundo aspecto radica en confundir las escalas de los procesos. Por ejemplo, no es raro que se confundan procedimientos con procesos o, peor aún, que se minimice la importancia de los programas como un conjunto de procesos. Sobre este tema resulta útil retroceder hasta los primeros años del siglo pasado; específicamente a la época en la que el afamado industrial Henry Ford aplicó la administración científica y los aportes de los padres de la administración, como el ingeniero y economista Frederick Taylor, para implementar el primer proceso de producción en serie (producción en masa) en la fabricación del automóvil Modelo T. Este vehículo marcó el inicio de la industria automovilística en una escala industrial para el consumo masivo y fue su producto estrella por más de una década.

El proceso de manufactura del Modelo T tenía la particularidad de producir un solo tipo de auto (el mismo diseño y únicamente de color negro). La aplicación de los principios de la administración científica y la tecnología de procesos de la época permitió implementar un solo proceso (gráfico 12) alimentado por un

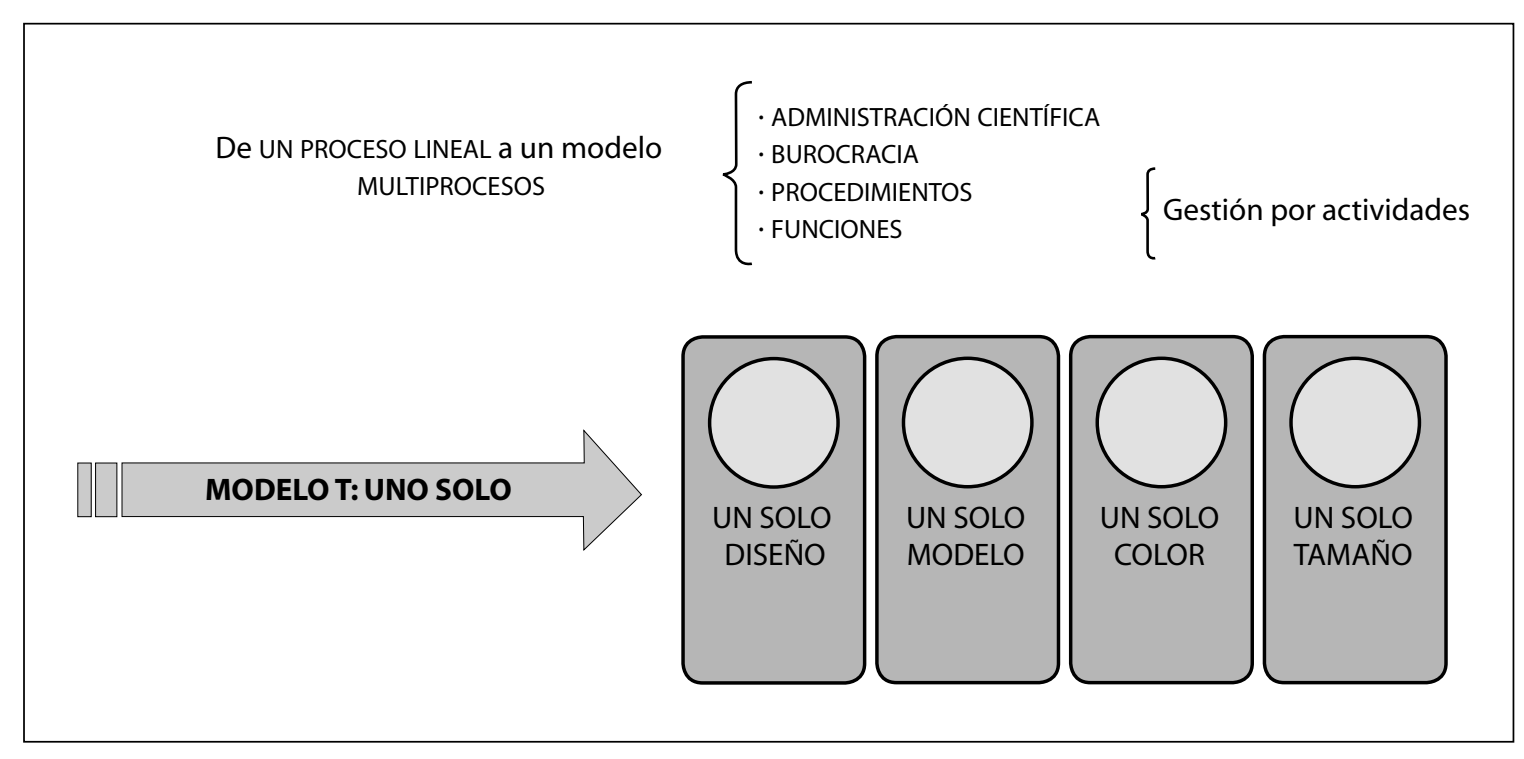

Gráfico 12

Modelo simple de gestión de procesos: un proceso lineal y muchas actividades y procedimientos 
elevado número de actividades, tareas y procedimientos automatizados y por una arquitectura organizativa jerárquico-funcional.

En los años siguientes, la competencia en el mercado, el crecimiento económico, el avance tecnológico y el cambio de los patrones de consumo, entre muchas otras tendencias que marcaron el auge de la era industrial y la competencia abierta en los mercados, determinaron la necesidad de introducir variantes al Modelo Ty ofrecer otros modelos. Henry Ford buscó también anticiparse a los cambios estructurales y transformó el modelo de gestión de un proceso lineal en un modelo de gestión multiprocesos, lo que coincidió con la adopción de una arquitectura organizativa más flexible.

Una lección de la experiencia narrada alude a privilegiar la escala, la temporalidad y la vigencia de los procesos. Respecto de la escala, todo proceso apunta a lograr un producto o un resultado que ha sido, de cierta forma, preestablecido; pero cuando el resultado o el producto tiene características que han variado o, más aun, un grado de complejidad considerablemente superior, entonces el mismo proceso ya no funcionará o, inclusive, es posible que se requiera operar más de un proceso para lograr el nuevo producto o resultado.

En cuanto a la temporalidad, todo proceso está sujeto a un ciclo de tres momentos: a) un momento en el cual los insumos que se van a emplear o la realidad que se va a transformar requieren prepararse, b) un momento en el cual las reglas del proceso actúan, apoyadas por una organización y un mecanismo de control y c) un momento en el cual los resultados se van produciendo y son perfectibles. Este ciclo requiere administrar el tiempo como variable y fijar puntos de control. No obstante, como se ha señalado, existe una inclinación a entender la temporalidad de los procesos como un ciclo indefinido, en el que no es práctico o factible implementar puntos de control.

Finalmente, sobre la vigencia, es importante notar que un proceso solo brinda los resultados esperados en la medida en que no existan nuevas versiones del proceso que ya hayan probado ser superiores y adaptarse mejor a la nueva realidad o las nuevas necesidades. Asimismo, la vigencia tiene mucho que ver con el arreglo institucional y el modelo de gestión que se combinan para gestionar los procesos; si este arreglo institucional falla, el proceso también fallará, inclusive si se hubiese actualizado.

La lógica sobre la gestión por procesos también opera en el campo gubernamental, aunque muchas veces esto no sea evidente para los gestores públicos. El primer paso es reconocer que una gestión desde lo local demanda no uno sino muchos procesos, todos ellos de alta complejidad, que requieren permanentemente de ciclos de corto, mediano y largo plazo y necesitan una organización inteligente y flexible pero, al mismo tiempo, muy ordenada.

El gráfico 13 busca reflejar un esquema de los procesos de gestión local. Con independencia de los tipos de competencia por operar (por ejemplo, temas de educación, salud, nutrición, ambiente, saneamiento, etcétera), los procesos nacen de una dimensión estratégica sustentada en los niveles operativo y organizativo: gestión por actividades y gestión por metas. Ambas dimensiones sirven para definir los macroprocesos y sus respectivos programas, proyectos, metas de desempeño, modelo de gestión en lo estrictamente funcional $y$ resultados.

Los gobiernos locales conducen procesos y también adaptan procesos que son, de cierto modo, estructurados; lo que significa que más de un gobierno local ejecuta procesos bajo un esquema similar de funcionamiento.

La gestión por procesos es, por definición, una gestión por resultados. La planificación estratégica, los procesos de fortalecimiento institucional y los proyectos de simplificación administrativa o modernización por sí solos no pueden dar resultados duraderos, y menos a costos razonables que garanticen la sostenibilidad de las mejoras.

Como se muestra en el cuadro 1, los insumos que forman parte de una gestión por procesos van mucho más allá de la dimensión administrativa y técnica y hallan su origen en un conjunto de decisiones desde múltiples sectores, con relación al estado presente local frente a lo global y a su proyección hacia el futuro. 
- ALIMENTACIÓN, DESNUTRICIÓN - SALUD, AGUA Y SANEAMIENTO

- EDUCACIONY CULTURA

- AMBIENTE Y SOSTENIBILIDAD
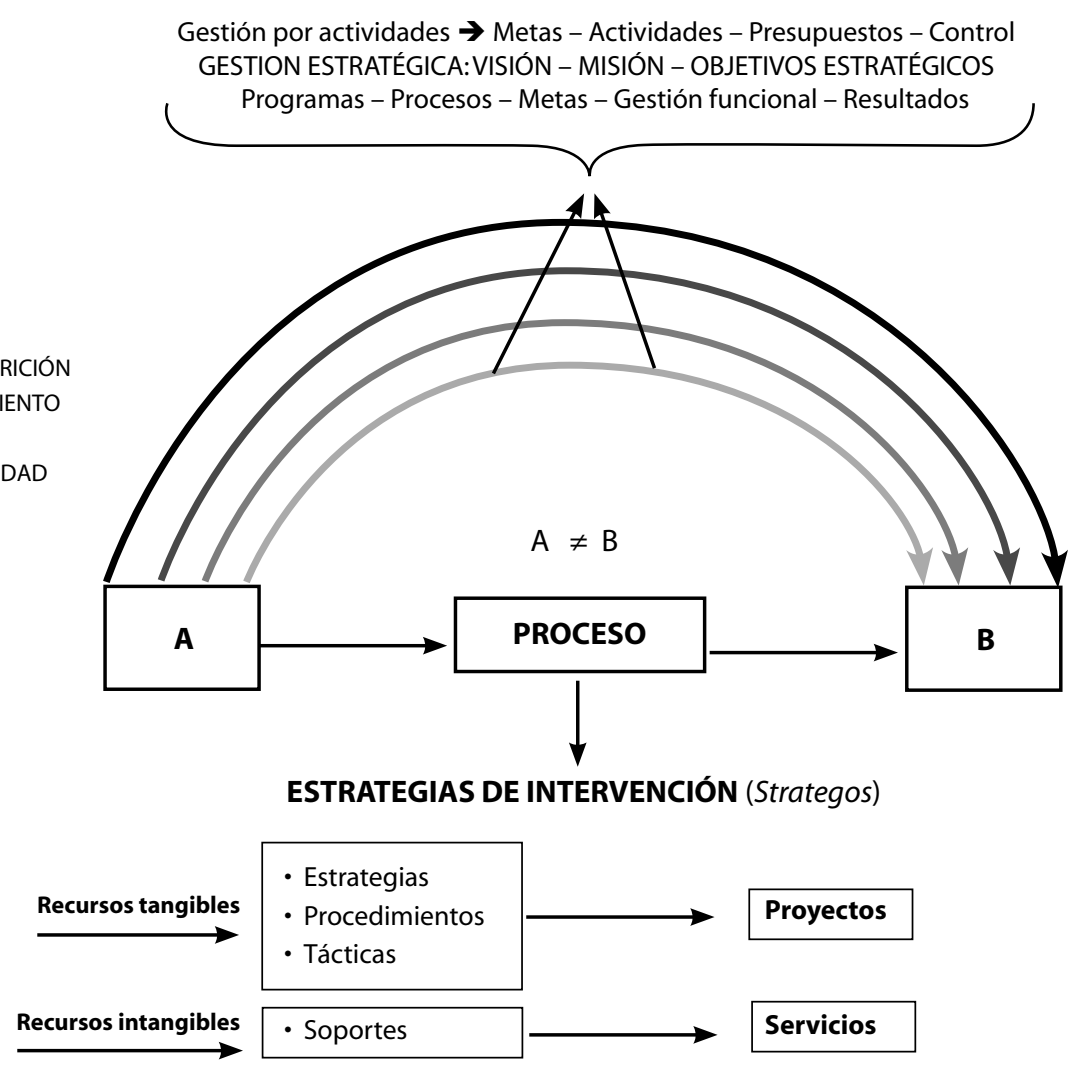

Gráfico 13

Configuración de procesos desde la gestión local

\section{Cuadro 1. Gestión por procesos orientada al control de la estrategia y la evaluación de resultados}

\begin{tabular}{|c|c|c|c|}
\hline INSUMOS & PROCESOS & RESULTADOS & TECNOLOGÍA \\
\hline $\begin{array}{l}\text { - Visión local } \\
\text { - Misión de los actores } \\
\text { - Situación local-externa }\end{array}$ & \multirow{3}{*}{ - Gestión multiproceso } & $\begin{array}{l}\text { - Programas de desarrollo } \\
\text { - Proyectos de impacto }\end{array}$ & \multirow{5}{*}{$\begin{array}{l}\text { - Tecnologías de la información } \\
\text { y toma de decisiones } \\
\text { - Tecnologías de gestión } \\
\text { - Tecnologías operativas } \\
\text { y administración de } \\
\text { operaciones }\end{array}$} \\
\hline $\begin{array}{l}\text { - Planificación estratégico-táctica } \\
\text { - Procesos participativos }\end{array}$ & & $\begin{array}{l}\text { - Servicios de calidad } \\
\text { - Control de la calidad }\end{array}$ & \\
\hline $\begin{array}{l}\text { - Recursos económicos y financieros } \\
\text { - Capacidades institucionales }\end{array}$ & & $\begin{array}{l}\text { - Formulación y articulación de programas } \\
\text { y proyectos }\end{array}$ & \\
\hline - Finanzas y rentas municipales & - Administración & - Gestión de los servicios públicos locales & \\
\hline $\begin{array}{l}\text { - Identificación de las áreas críticas para } \\
\text { su ejecución }\end{array}$ & $\begin{array}{l}\text { - Diseño de estrategias } \\
\text { - Reingeniería de procesos }\end{array}$ & $\begin{array}{l}\text { - Gestión de programas y proyectos en } \\
\text { ejecución }\end{array}$ & \\
\hline
\end{tabular}


Dentro de este ciclo, las políticas y las decisiones desde el gobierno local solo son uno de los componentes desde el punto de vista de la gobernabilidad local, pues los roles y las expectativas de los demás actores son tan o más importantes.

En un nivel inmediatamente inferior, los insumos comprenden los procesos de planificación (estrategia) y el diseño organizacional que soporta a lo planificado (estructura), el grado de complementariedad de recursos y capacidades institucionales e intersectoriales, la gerencia financiera y la capacidad técnica y tecnológica. Todos estos insumos son «el objeto de la transformación»; en consecuencia, el proceso integral de transformación se denominará «la gestión»e incluirá muchos procesos, la administración de la estructura y el diseño de estrategias de fortalecimiento institucional y mejora o ajuste de procesos (reingeniería).

Como parte de la gestión de procesos, el logro de resultados es la meta suprema. Esta afirmación parece contradecir lo antes descrito y enfatizado (ver cuadro 1), puesto que el modelo de gestión por procesos, según se afirmó, tiene el propósito de alinear el accionar y las decisiones de la organización a los componentes de la dimensión estratégica de la gestión. Sin embargo, si los más altos valores y la visión de la organización no se pueden alcanzar, o son solo buenas intenciones, ocurre algo paradójico: se tienen procesos alineados con la principal estrategia de la organización pero esta no es buena o es irrealizable.

Si los procesos son efectivos y su gestión también, el conjunto debe acercar a la organización a los resultados esperados; pero si falla el diseño de los procesos o su gestión no habrá control alguno sobre la medición del logro de los resultados. Esto confirma que los resultados se obtienen en función de procesos siempre que los procesos estén alineados con la principal estrategia de la organización y que esta estrategia sea suficientemente sólida y posible. Si no hay articulación de estas dimensiones no es posible avanzar en la implementación de un modelo de gestión ni gestionar los procesos de modo sostenido (gráfico 14).

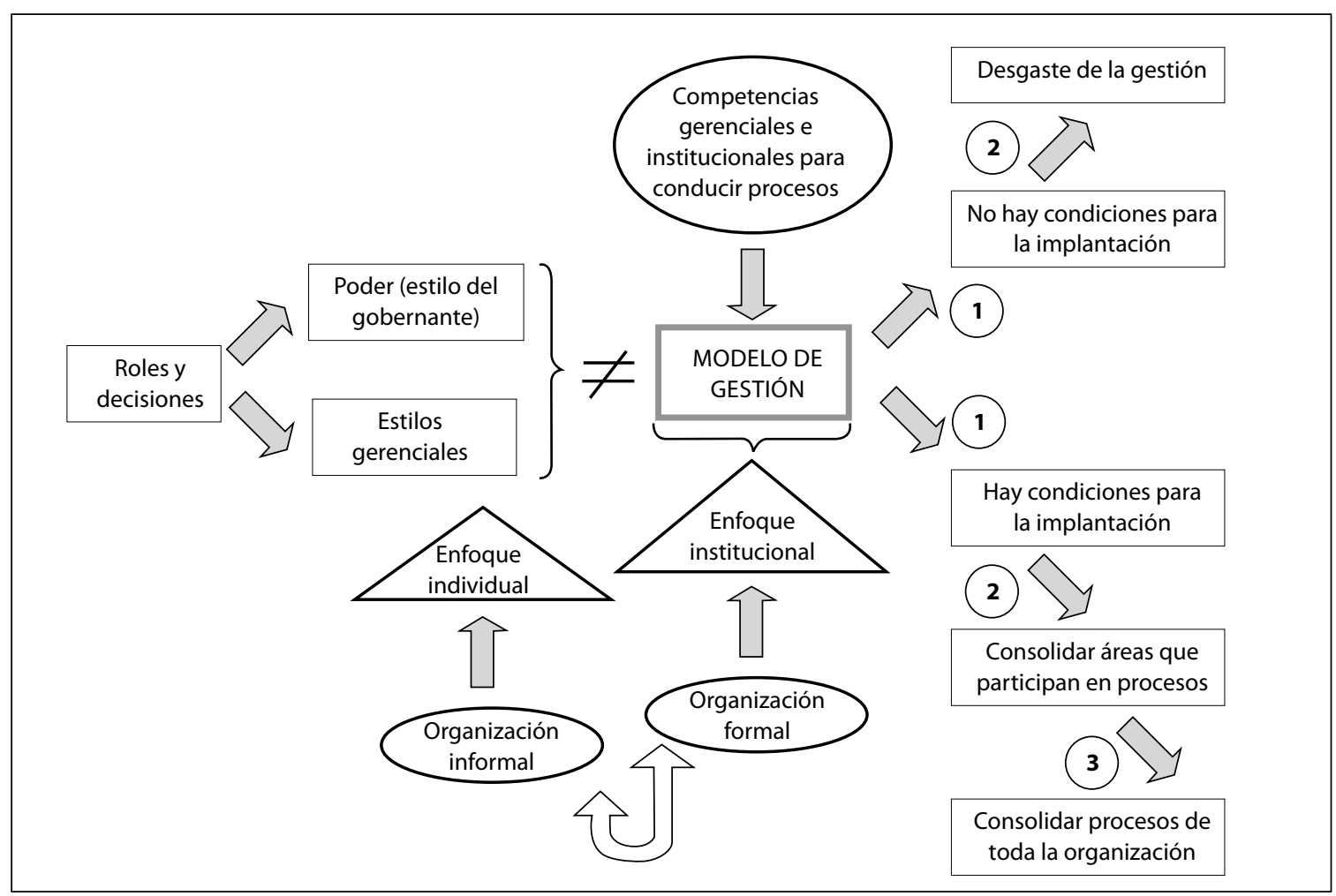

Gráfico 14

Modelo de gestión, gestión por procesos y dirección de organizaciones 
El gráfico 14 ilustra los distintos componentes que gravitan en torno a la adopción de un modelo de gestión por procesos. La orientación de este modelo es, por un lado, hacia equilibrar las dimensiones de la estructura y la estrategia de la organización y, por el otro, a alinear los procesos a la principal estrategia de la organización de modo que su gestión se consolide progresivamente, no solo en el nivel de la toma de decisiones sino también en el de las dimensiones más operativas dentro de la organización, incluyendo los procedimientos y las operaciones que tienen a su cargo las áreas administrativas y de apoyo.

Una gestión por procesos requiere que la organización posea condiciones para su implantación. Si la gestión está sesgada hacia los enfoques de los individuos con poder para tomar decisiones, sus «estilos» van a impedir que la visión técnica sea compatible con la visión política, por eso es un hecho político-técnico la necesidad de adoptar un modelo de gestión. Con igual criterio, pero en un nivel operativo, es esencial entender cómo funciona la organización en su dimensión informal, lo que se asocia a la cultura y el comportamiento organizacional. Esta debe ser analizada para estimular que la adopción del modelo de gestión se independice de los sesgos, las aversiones y las preferencias de las personas y pueda ser institucionalizada.

Además, se requiere de un considerable periodo de pruebas, no solo porque el sistema nuevo, al ser integral, demanda de manera sucesiva (en frío y en caliente) pruebas, ajustes $y$, eventualmente, actualizaciones, sino porque no es de esperar que en la organización existan todas las competencias gerenciales y las capacidades institucionales para llevar adelante el proceso de implantación. Esta curva de aprendizaje debe adquirirse y ser puesta a prueba en condiciones reales, es decir, a través del mismo proceso, para desarrollar condiciones para la implantación (paso 1), la consolidación del modelo como proceso piloto (paso 2) y la consolidación de los procesos y el modelo en toda la organización (paso 3). De no existir las condiciones de implantación, eventualmente la gestión se desgastará y las mejoras no se producirán.

En conclusión, todo proceso de modernización o fortalecimiento, por ejemplo la implantación de un mo- delo de gestión por procesos orientados a maximizar el desempeño o la productividad de la organización, requerirá tener en cuenta los principios descritos.

\section{CONTRIBUCIONES Y LIMITACIONES}

La investigación realizada sugiere que es en virtud a conciliar los supuestos de las dos grandes escuelas del proceso estratégico (la escuela prescriptiva y la escuela descriptiva) que es posible generar el sustrato para aplicar los fundamentos del control estratégico y alentar la adopción de tecnologías de gestión para la evaluación del desempeño en las organizaciones del sector público; particularmente en el caso de los gobiernos locales, que es el campo de estudio empleado por los autores para el desarrollo de la herramienta descrita. Este aspecto también constituye una limitación del estudio presentado en forma parcial en este artículo, dado que el diseño ha sido influenciado tanto por la complejidad de la gestión pública local como por las características de las políticas y los procesos de descentralización y modernización del Estado en curso en la escena peruana. Una variación sustancial de los escenarios que sirvieron de sustrato al diseño de la herramienta podría implicar ajustes al modelo planteado.

El enfoque estructura-estrategia adoptado tiene como base criterios sobre el valor agregado que el proceso de gestión aporta a las organizaciones y que subyacen tanto a las teorías claves sobre las organizaciones, la firma, los sistemas y los procesos como reflejo del funcionamiento de sistemas dinámicos, como al diseño de tecnologías de gestión para medir el desempeño de la firma como: administración por objetivos, modelos de gestión de la calidad (como el Malcolm Baldrige), cuadros de mando integral (como el Balanced Scorecard), estimación económica del valor agregado (EVA) y gerencia basada en valor (VBM), entre otros.

No obstante, el enfoque estructura-estrategia adoptado no logra identificarse como un medio para hacer extensivo al sector gubernamental el empleo de los desarrollos teóricos e instrumentales en materia de control estratégico del sector empresarial. Un esfuerzo de tal envergadura no solo excede los alcances 
del presente trabajo, sino que además demanda un estudio profundo sobre el aporte de los modelos de gestión basados en enfoques mecanicistas al desempeño de organizaciones del ámbito de la gestión gubernamental o pública.

El eje de esta propuesta se centra en el reconocimiento de la gestión como un proceso que, por definición, existe y subsiste en la medida en que los objetivos de gestión se definan, los resultados que reflejen el logro de metas y objetivos en un periodo razonable de tiempo también estén predefinidos y el sistema de toma de decisiones se apoye en un conjunto de medidas pensadas para controlar la marcha de la estrategia y, por tanto, articular objetivos, decisiones, uso de recursos y evaluación de metas de logro. En consecuencia, la propuesta ha permitido derivar un modelo general para conducir el proceso de gestión bajo parámetros flexibles que pueden complementar en buena medida los roles funcionales desde las diversas áreas de una organización, lo que permite que el esquema propuesto sea aplicado a instituciones de gobierno local (municipalidades).

No obstante, eventuales esfuerzos de implementación demandarán el desarrollo complementario de nuevas aproximaciones teóricas y metodológicas en campos como la arquitectura institucional, la competitividad territorial, los límites en el ejercicio de competencias de la organización gubernamental (de modo similar a lo que se conoce como boundaries of the firm), procesos de fortalecimiento institucional, gestión de los recursos humanos bajo parámetros de medición del desempeño, un renovado marco normativo y gestión del cambio y la resistencia dentro de las organizaciones, entre otros aspectos que se deben enfrentar para intentar reducir la brecha en los desarrollos intelectuales y prácticos que marcan distancias entre la gestión estratégica en los ámbitos empresarial y gubernamental.

\section{Referencias bibliográficas}

Ansoff, I. \& McDonnell, E. (1990). Implanting strategic management. Londres: Prentice Hall.

Arrow, K. (1974). The limits of the organization. Nueva York, NY: Norton.

Barzelay, M. (2000). The new public management. Berkeley, CAL: University of California Press.

Becerra, M. (2009). Theory of the firm for strategic management. Economic value analysis. Cambridge: Cambridge University Press.

Chandler, A. D. (1962). Strategy and structure: Chapters in the history of the American industrial enterprise. Cambridge-MIT Institute, MA: MIT Press.

Chandler, A. D. (1977). The visible hand:The managerial revolution in American business. Cambridge, MA / Londres: The Belknap Press of Harvard University Press.

Chenhall, R. H. (2005). Content and process approaches to studying strategy and management control systems. En Chapman, C. S. (ed.), Controlling strategy: Manage- ment, accounting and performance measurement. Nueva York, NY: Oxford University Press.

Clegg, S. R., Courpasson, D. \& Phillips, N. (2006). Power and organizations. Londres:Sage.

Coase, R. H. (1937, noviembre). The nature of the firm. Economica, 4, 386-405.

Duck, D. J. (2001). Gestionar el cambio: el arte del equilibrio. En Harvard Business Review (ed.), Gestión del cambio (pp. 73-104). Bilbao: Deusto.

Galbraith, J. R. (1973). Designing complex organizations. Boston, MA: Addison-Wesley / Longman.

Gouldner, A. W. (1964). Patterns of industrial bureaucracy. Nueva York, NY:The Free Press.

Joyce, P. (2001). Strategic management for public services. Buckingham / Philadelphia, PA:Open University Press.

Kaplan, R. \& Norton, D. P. (1993, septiembre-octubre). Putting the balanced scorecard to work. Harvard Business Review, 71 (5), 134-147. 
Kaplan, R. S. \& Norton, D. P. (1996). The balanced scorecard: Translating strategy into action. Boston, MA: Harvard Business School Press.

Kaplan, R. S. \& Norton, D. P. (2001). The strategy-focused organization: How balanced scorecard companies thrive in the new business environment. Boston, MA: Harvard Business School Press.

Kaufman, R. (2000). Mega planning: Practical tools for organizational success. Thousand Oaks, CAL: Sage.

Kenny, G. (2005). Strategic planning and performance management: Develop and measure a winning strategy. Burlington, MA: Elsevier Butterworth-Heinemann.

Kotter, P. J. (2001). Liderar el cambio: por qué fracasan los intentos de transformación.En Harvard Business Review (ed.), Gestión del cambio (pp. 7-20). Bilbao: Deusto.

Lawrence, P. \& Lorsch, J. (1967). Differentiation and integration in complex organizations. Administrative Science Quarterly, 12, 1-30.

Leigh, D. (2003, junio). Worthy performance, redux. Performance Xpress. International Society for Performance Improvement Newsletter. $<$ http://www.performancexpress.org/0306.

Longo, F. (2000). La nueva gestión pública en la reforma del núcleo estratégico del gobierno: experiencias latinoamericanas. Caracas: Centro Latinoamericano de Administración para el Desarrollo (CLAD). $\measuredangle t$ ttp://www.iadb. org/sds/doc/sgc-estudioCLAD.pdf .

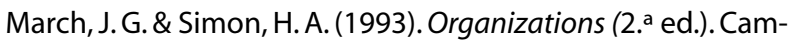
bridge, MA: Blackwell.

Metcalfe, L. \& Richards, S. (1987). Improving public management. London: Sage.
Mintzberg, H. (1989). Mintzberg on management: Inside our strange world of organizations. Nueva York, NY: The Free Press.

Mintzberg, H., Ahlstrand, B. \& Lampel, J. (1998). Strategy safari: A guided tour through the wilds of strategic management. Nueva York, NY:The Free Press.

Oakland, J. S. (2004). Oakland in quality management. Burlington, MA: Elsevier Butterworth-Heinemann.

Porter, M. E. (1998). Competitive advantage: Creating and sustaining superior performance. Nueva York, NY:The Free Press.

Quinn, R. E., Farman, S. R., Thompson, M. P. \& McGrath, M. R. (2003). Becoming a master manager. A competency framework. Nueva York, NY: John Wiley \& Sons.

Robbins, S. \& Coulter, M. (1996). Administración. México, D. F.: Prentice Hall.

Sabattini, L. \& Crosby, F. (2008). Overcoming resistance: Structures and attitudes. En K. M: Thomas (ed.), Diversity resistance in organizations. Nueva York, NY: Lawrence Erlbaum.

Taylor, F. W. (1911). The principles of scientific management. Nueva York, NY: Harper \& Brothers.

Tirole, J. (1994). The theory of industrial organization. Cambridge, MA:The MIT Press.

Watkins, R. (2007). Performance by design. The systemic selection, design, and development of performance technologies that produce useful results. Amherst, MA:HRD Press.

Weber, M. C. E. (1947). The theory of social and economic organization. Nueva York, NY: Oxford University Press. 